\title{
Prospects for the Measurement of the Structure of the Coupling of a Higgs Boson to Weak Gauge Bosons in Weak Boson Fusion with the ATLAS Detector
}

\author{
Christoph Ruwiedel ${ }^{1}$, Markus Schumacher ${ }^{2}$, Norbert Wermes ${ }^{1}$ \\ ${ }^{1}$ Physikalisches Institut, Universität Bonn, Germany \\ ${ }^{2}$ Fachbereich Physik, Universität Siegen, Germany
}

May 2, 2007

\begin{abstract}
The prospects for the measurement of the tensor structure of the vertex between a standard model Higgs boson and two weak gauge bosons using the distribution of the azimuthal angles between the two tagging jets in the weak boson fusion channel are studied in a Monte Carlo analysis using the fast simulation of the ATLAS detector. The decay channels $H \rightarrow \tau^{+} \tau^{-} \rightarrow l l+4 \nu, H \rightarrow \tau^{+} \tau^{-} \rightarrow l h+3 \nu$ at $m_{H}=120 \mathrm{GeV}$ and $H \rightarrow W^{+} W^{-} \rightarrow l l \nu \nu$ at $m_{H}=160 \mathrm{GeV}$ are used in the analysis. For a standard model Higgs boson it is found that purely anomalous couplings are expected to be excluded at a confidence level corresponding to $2 \sigma$ or more at $m_{H}=120 \mathrm{GeV}$ and more than $5 \sigma$ at $m_{H}=160 \mathrm{GeV}$ from $30 \mathrm{fb}^{-1}$ of data. With a value of 1 roughly reproducing the standard model cross section for a purely anomalous coupling, the standard deviation in a measurement of a contribution of a $\mathrm{CP}$ even anomalous coupling in addition to the standard model coupling is estimated to be 0.20 at $m_{H}=120 \mathrm{GeV}$ and 0.09 at $m_{H}=160 \mathrm{GeV}$.
\end{abstract}

\section{Introduction}

One of the main purposes of the ATLAS experiment is the investigation of the predicted effects of electroweak symmetry breaking and the description of particle masses in the standard model. Monte Carlo studies of the discovery potential for a standard model Higgs boson, which is a necessary component of the symmetry breaking mechanism of the standard model, indicate that it will be possible to discover the Higgs boson, if it exists, over the whole mass range which is not yet excluded from measurements of the electroweak sector. One of the most promising channels for the discovery of a Higgs boson is the weak boson fusion channel[1].

After the discovery of a new particle at the LHC the question will arise what kind of particle it is. Thus, in a next step, its quantum numbers and couplings will be investigated. It has been proposed [2] to use the distribution of the azimuthal angles between the tagging jets in weak boson fusion to determine the structure of the coupling of a scalar Higgs particle candidate to two weak bosons. For simplicity, the scalar particle under study will sometimes 
just be called Higgs boson in the following. The potential for this measurement with the ATLAS detector is studied using the fast detector simulation ATLFAST. The angular correlations that are used are largely independent of the mass and decay channel of the Higgs boson. Thus, the analysis can be performed for different decay channels and Higgs boson masses in a similar way.

In addition to the standard model coupling of a Higgs boson to weak gauge bosons anomalous couplings arising from terms in an effective Lagrangian are considered. A measurement of the anomalous couplings can provide information about the CP quantum number of the Higgs boson and about possible loop contributions to the coupling from particles with large mass.

The structure of the coupling vertex with standard model and anomalous contributions is discussed in section 2. The present experimental limits on the anomalous couplings from measurements at LEP and especially the L3 experiment are given in section 2.2.

Event generation and event selection are discussed in sections 3 and 4 .

The first of two parts of the analysis, an investigation of the prospects for the determination of the dominant coupling term, is presented in section 5. This measurement can be used to determine the $\mathrm{CP}$ quantum number of the scalar particle and it can be used to decide whether the coupling is loop-induced or standard model-like. The case of a standard model Higgs boson for which anomalous couplings are to be excluded is studied as a reference case in this section.

In the second part of the analysis the sensitivity of the ATLAS experiment to a contribution by an anomalous coupling in addition to the standard model coupling is estimated using a likelihood fit. The results of this analysis are presented in section 6 .

This note is based on the results of [3] (in German).

\section{Anomalous Higgs boson couplings}

Deviations from the predictions of the standard model for the couplings of a Higgs boson to weak gauge bosons are parametrised in a systematic and model-independent way in an effective, gauge invariant Lagrangian. This effective Lagrangian contains terms composed of Higgs and weak gauge fields with mass dimension higher than 4. A theory containing such terms is not renormalisable. It is considered an effective theory that is valid only up to a certain energy scale $\Lambda$ where it has to be replaced by a more fundamental theory containing new particles. An effective Lagrangian is often written as an expansion in $1 / \Lambda$ :

$$
\mathcal{L}_{e f f}=\mathcal{L}_{S M}+\sum_{i} \frac{g_{i}^{(5)}}{\Lambda} \mathcal{O}_{i}^{(5)}+\sum_{i} \frac{g_{i}^{(6)}}{\Lambda^{2}} \mathcal{O}_{i}^{(6)}+\ldots
$$

In this expansion the operators are ordered by their dimension and are assumed to contribute less the higher their dimension is. The $g_{i}$ are dimensionless coupling constants with a magnitude of the order of 1 . A comprehensive list of operators of up to dimension 6 is given in [4]. In this study, the following operators are considered, as given in [5]:

$$
\begin{aligned}
\mathcal{L}_{5}= & \frac{g_{5 e}^{H W W}}{\Lambda_{5 e}} H W_{\mu \nu}^{+} W^{-\mu \nu}+\frac{g_{5 o}^{H W W}}{\Lambda_{5 o}} H \tilde{W}_{\mu \nu}^{+} W^{-\mu \nu}+ \\
& \frac{g_{5 e}^{H Z Z}}{2 \Lambda_{5 e}} H Z_{\mu \nu} Z^{\mu \nu}+\frac{g_{5 o}^{H Z Z}}{2 \Lambda_{5 o}} H \tilde{Z}_{\mu \nu} Z^{\mu \nu}
\end{aligned}
$$

The dimension 5 operators in equation (2) arise from dimension 6 operators of the structure $\left(\Phi^{\dagger} \Phi\right) V_{\mu \nu} V^{\mu \nu}$ and $\left(\Phi^{\dagger} \Phi\right) \tilde{V}_{\mu \nu} V^{\mu \nu}$, with $V=W / Z$, when the Higgs field is written in its 
physical expansion,

$$
\phi=\frac{1}{\sqrt{2}}\left(\begin{array}{c}
0 \\
v+H(x)
\end{array}\right) .
$$

The field strength tensor $V^{\mu \nu}$ of the field $V$ is even under CP transformations while the dual field strength tensor, given by

$$
\tilde{V}^{\mu \nu}=\frac{1}{2} \epsilon^{\mu \nu \rho \sigma} V_{\rho \sigma},
$$

is $\mathrm{CP}$ odd.

As long as the field $H$ in equation (2) is the standard model Higgs boson field, which is $\mathrm{CP}$ even, the operators composed of the field strength tensors are $\mathrm{CP}$ even, indicated by the subscript $e$ on the coupling constant $g$ and the scale $\Lambda$, while the operators containing the dual field strength tensors are $\mathrm{CP}$ odd, indicated by the subscript $o$. In a CP conserving theory only the $\mathrm{CP}$ even operators will contribute to the coupling. In more general models with additional scalar particles the effective Lagrangian in equation (2) may be used to describe the couplings of those scalar particles to weak gauge bosons. If in this case $H$ represents a CP odd scalar particle, the operators will switch their behaviour under CP transformations. The operators containing the dual field strength tensors will now be CP even and give the only contribution to the coupling if $\mathrm{CP}$ is conserved. Thus, assuming CP conservation it is possible to determine the $\mathrm{CP}$ quantum number of the scalar particle by experimentally distinguishing between the coupling terms. This is one of the goals of the analysis presented in section 5 .

The vertex that couples a Higgs boson to vector bosons is written in its most general form as [5]:

$$
\begin{aligned}
T^{\mu \nu}\left(q_{1}, q_{2}\right)= & a_{1}\left(q_{1}, q_{2}\right) g^{\mu \nu}+a_{2}\left(q_{1}, q_{2}\right)\left[q_{1} \cdot q_{2} g^{\mu \nu}-q_{2}^{\mu} q_{1}^{\nu}\right]+ \\
& a_{3}\left(q_{1}, q_{2}\right) \epsilon^{\mu \nu \rho \sigma} q_{1 \rho} q_{2 \sigma}
\end{aligned}
$$

with the four-momenta of the vector bosons $q_{1}$ and $q_{2}$. The $a_{i}\left(q_{1}, q_{2}\right)$ are Lorentz-invariant form factors. In the standard model at leading order, the only non-vanishing coefficient is given by:

$$
a_{1}\left(q_{1}, q_{2}\right)=\frac{2 m_{V}^{2}}{v}
$$

The effective Lagrangian of equation 2 gives the additional couplings

$$
a_{2}\left(q_{1}, q_{2}\right)=-\frac{2}{\Lambda_{5 e}} g_{5 e}^{H W W}, \quad a_{3}\left(q_{1}, q_{2}\right)=\frac{2}{\Lambda_{5 o}} g_{5 o}^{H W W}
$$

for the $H W W$ vertex and

$$
a_{2}\left(q_{1}, q_{2}\right)=-\frac{2}{\Lambda_{5 e}} g_{5 e}^{H Z Z}, \quad a_{3}\left(q_{1}, q_{2}\right)=\frac{2}{\Lambda_{5 o}} g_{5 o}^{H Z Z}
$$

for the $H Z Z$ vertex.

As in [5], the ratio of the anomalous $H W W$ couplings to the anomalous $H Z Z$ couplings is chosen according to $g_{5 e / o}^{H W W}=g_{5 e / o}^{H Z Z} \cos ^{2} \theta_{w}$ in this analysis which corresponds to a ratio equal to that of the respective standard model couplings. The scale $\Lambda_{5 e / o}$ is fixed, as in [5], to a value of $480 \mathrm{GeV}$ which roughly reproduces the standard model cross section for weak boson fusion at a Higgs boson mass of $120 \mathrm{GeV}$ and for a purely anomalous CP even or a purely anomalous CP odd coupling with $g_{5 e / o}^{H W W}=g_{5 e / o}^{H Z Z} \cos ^{2} \theta_{w}=1$. This leaves two free parameters, e.g. $g_{5 e}^{H Z Z}$ and $g_{5 o}^{H Z Z}$, which define the strength of the anomalous couplings. 


\subsection{Examples for Anomalous Higgs-vector boson Couplings}

A well-known example for the occurrence of the CP even anomalous coupling is the production of a Higgs boson in the standard model through gluon-gluon-fusion, which is mediated by a top quark-dominated loop. In the limit of a large top quark mass the vertex is of the form $T^{\mu \nu} \propto\left(q_{1} \cdot q_{2} g^{\mu \nu}-q_{1}^{\nu} q_{2}^{\mu}\right)$ with the momenta of the gluons $q_{1}$ and $q_{2}$. The similar loop-induced contribution to the coupling of a Higgs boson to weak bosons in the standard model is suppressed by a factor of $\alpha_{W} / \pi \approx 10^{-2}$ [2]. It will be shown in section 6 that a contribution of this size will not be observable at the LHC.

The CP odd $A^{0}$ Higgs boson of the minimal supersymmetric standard model does not couple to weak bosons at leading order. There is an anomalous loop-induced coupling of the form $A^{0} \tilde{V}_{\mu \nu} V^{\mu \nu}$ which, however, will be too weak to be observable at the LHC. The effective coupling of a top-pion, which occurs in topcolor models, to weak bosons is cited in [2] as an example of a coupling described by the operators containing the dual field strength tensors which may lead to an observable event rate at the LHC.

\subsection{Experimental Limits on the Anomalous Couplings}

The possibility of a contribution of a CP even anomalous coupling of a Higgs boson to weak gauge bosons in addition to the standard model coupling has been studied at LEP by the L3 collaboration. The resulting limits on the couplings have been published in [6]. In the parametrisation of the L3 collaboration, the relevant part of the effective Lagrangian is:

$$
\begin{aligned}
\mathcal{L}_{e f f}= & g_{H W W}^{(1)}\left(W_{\mu \nu}^{+} W_{-}^{\mu} \partial^{\nu} H+\text { h.c. }\right)+g_{H W W}^{(2)} H W_{\mu \nu}^{+} W_{-}^{\mu \nu} \\
& +g_{H Z Z}^{(1)} Z_{\mu \nu} Z^{\mu} \partial^{\nu} H+g_{H Z Z}^{(2)} H Z_{\mu \nu} Z^{\mu \nu}
\end{aligned}
$$

where

$$
\begin{aligned}
g_{H W W}^{(1)} & =\frac{g m_{W}}{m_{Z}^{2}} \Delta g_{1}^{Z} \\
g_{H W W}^{(2)} & =\frac{g}{m_{W}} d \\
g_{H Z Z}^{(1)} & =\frac{g}{m_{W}}\left(\Delta g_{1}^{Z} \cos 2 \theta_{W}+\Delta \kappa_{\gamma} \tan ^{2} \theta_{W}\right) \\
g_{H Z Z}^{(2)} & =\frac{g}{2 m_{W}}\left(d \cos ^{2} \theta_{W}+d_{B} \sin ^{2} \theta_{W}\right)
\end{aligned}
$$

All of the terms in equation (9) give contributions to the CP even anomalous coupling $a_{2}$ of equation (5) even though in this parametrisation not all of the terms are of the form $H V_{\mu \nu} V^{\mu \nu}$. Limits on CP odd anomalous couplings were not determined in the L3 study.

The limits on the parameters $d$ and $d_{B}$ from the L3 study are given as the $95 \%$ confidence level contour plots shown in figure 1. For the Higgs boson mass values used in this analysis the limits were read from the plots and collected in table 1. The parameters $\Delta g_{1}^{Z}$ and $\Delta \kappa_{\gamma}$ also appear in parametrisations of deviations from the standard model triple gauge boson couplings and the best limits were established from measurements of triple gauge boson couplings by all four LEP experiments. The limits on these parameters were determined from values published in [23] and collected in table 2 .

Values of the parameters $d, d_{B}, \Delta g_{1}^{Z}$ and $\Delta \kappa_{\gamma}$ are converted to corresponding values of the parameters $g_{5 e}^{H W W}$ and $g_{5 e}^{H Z Z}$ of equation (2) using the following conversion rules from the program vbfnlo (see section 3.1 ):

$$
g_{5 e}^{H W W}=\Lambda_{5 e} \frac{g}{m_{W}}\left(d+\Delta g_{1}^{Z} \frac{m_{W}^{2}}{m_{Z}^{2}}\right)
$$


Exclusion (95\% CL):

$\square$ Combined $\quad \square$ ee $\rightarrow$ rry, eer $\square$ ee $\rightarrow \mathrm{HZ}$

-- Expected limit

ee $\rightarrow$ Zry

ee $\rightarrow \mathrm{WW}^{(*)} \gamma$
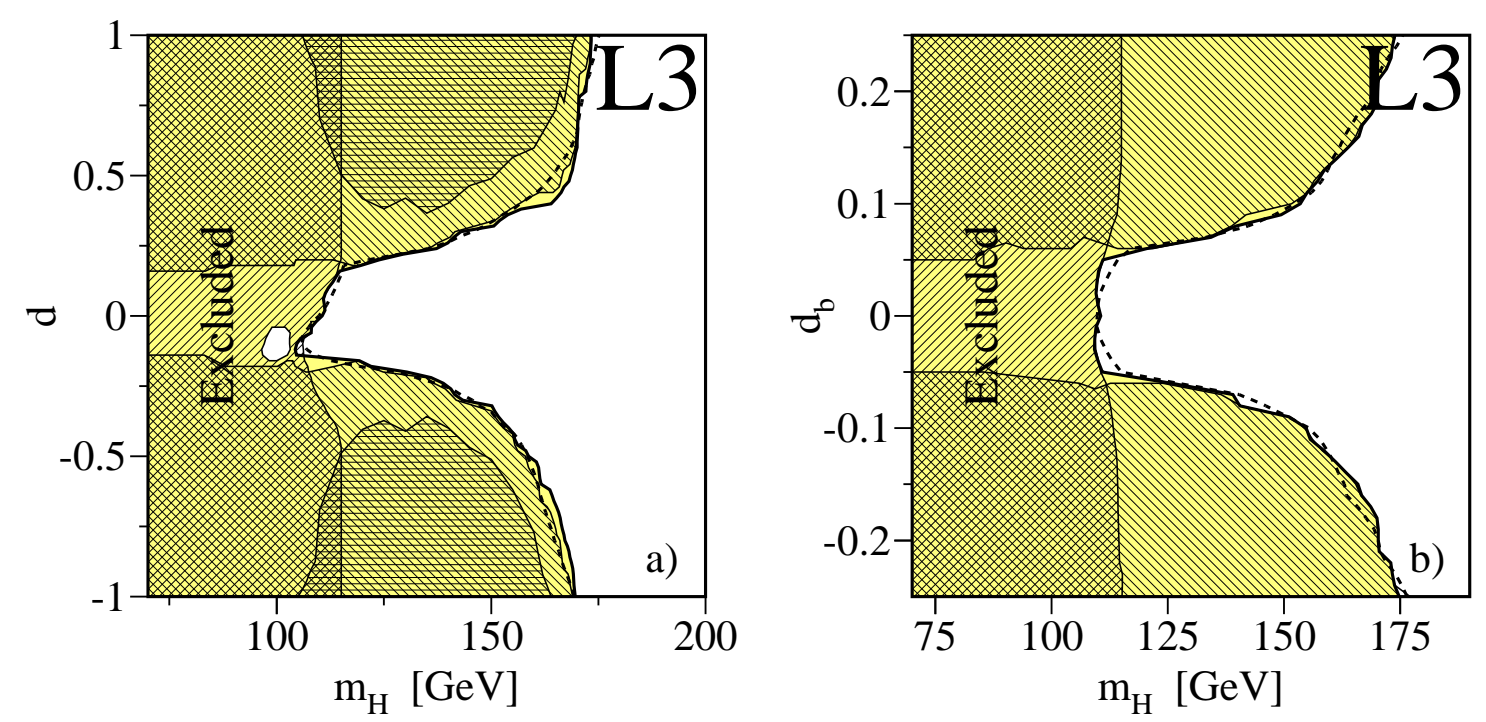

Figure 1: $95 \%$ confidence level (CL) exclusion limits on the parameters $d$ and $d_{B}$ as published by the L3 collaboration [6].

\begin{tabular}{|ccc|}
\hline$m_{H}$ & $d$ & $d_{B}$ \\
\hline $120 \mathrm{GeV}$ & $-0.19 \ldots 0.19$ & $-0.06 \ldots 0.06$ \\
$160 \mathrm{GeV}$ & $-0.50 \ldots 0.40$ & $-0.13 \ldots 0.14$ \\
\hline
\end{tabular}

Table 1: $95 \%$ confidence level intervals for the parameters $d$ and $d_{B}$, determined from the plots in figure 1.

\begin{tabular}{|cc|}
\hline$\Delta g_{1}^{Z}$ & $\Delta \kappa_{\gamma}$ \\
\hline$-0.051 \ldots 0.034$ & $-0.105 \ldots 0.069$ \\
\hline
\end{tabular}

Table 2: $95 \%$ confidence level intervals for the parameters $\Delta g_{1}^{Z}$ and $\Delta \kappa_{\gamma}$, taken from [23].

\begin{tabular}{|ccc|}
\hline$m_{H}$ & $g_{5 e}^{H W W}$ & $g_{5 e}^{H Z Z}$ \\
\hline $120 \mathrm{GeV}$ & $-0.78 \ldots 0.73$ & $-0.63 \ldots 0.55$ \\
$160 \mathrm{GeV}$ & $-2.0 \ldots 1.5$ & $-1.6 \ldots 1.3$ \\
\hline
\end{tabular}

Table 3: Approximate $95 \%$ confidence level intervals for the parameters $g_{5 e}^{H W W}$ and $g_{5 e}^{H Z Z}$, calculated from the values in tables 1 and 2 (see text). 


$$
g_{5 e}^{H Z Z}=\Lambda_{5 e} \frac{g}{m_{W}}\left(d \cos ^{2} \theta_{W}+d_{B} \sin ^{2} \theta_{W}+\Delta g_{1}^{Z} \cos 2 \theta_{W}+\Delta \kappa_{\gamma} \tan ^{2} \theta_{W}\right)
$$

Rough estimates of $95 \%$ confidence level intervals for the parameters $g_{5 e}^{H W W}$ and $g_{5 e}^{H Z Z}$ were determined by using error propagation in equations (14) and (15) which implies the assumption that the parameters $d, d_{B}, \Delta g_{1}^{Z}$ and $\Delta \kappa_{\gamma}$ are uncorrelated and distributed according to Gaussian distributions around the central values of the confidence intervals. If the parameters are correlated to some degree the approximation should still be reasonable because the resulting limits are dominated by the uncertainty on the parameter $d$. For the purpose of comparing the results of this study to the limits from the L3 analysis the calculated limits can be regarded as conservative in the sense that they may overestimate the sensitivity of the L3 study. The limits are given in table 3 .

One can see from the values given in table 3 that the limits on the Higgs boson coupling to $W$ boson pairs are slightly weaker than the limits on the coupling to $Z$ boson pairs. One can also see from figure 1 and from tables 1 and 3 that the limits on the anomalous couplings get weaker as the Higgs boson mass increases.

\section{Event Generation}

Events for some of the processes used in this study were generated with parton level event generators while others were generated with Pythia. The events from the parton level generators were passed to the ATLAS software framework ATHENA using data files containing the information from the Les Houches common blocks [7]. Parton shower, fragmentation, hadron decays, underlying event, $\tau$ lepton decays, final state electromagnetic radiation from $\tau$ leptons and the detector simulation were calculated for all processes with Pythia 6.226 [8], Tauola 2.7 [9], Photos 2.6 [10] and ATLFAST [11] respectively. Version 10.0.1 of ATHENA was used. The version of ATLFAST used in ATHENA 10.0.1 has not been formally validated within the ATLAS collaboration and, albeit being considered as appropriate for this study, results obtained using this version cannot be compared directly to other ATLAS results. In all programs the parton density functions CTEQ5L [12] were used.

For the reconstruction of jets in ATLFAST a basic simulation of the calorimeter is used. Particle energies are summed in cells from which then clusters are formed following a conebased method with a cone radius of $\Delta R=0.4^{1}$. The energies of the clusters are then modified using a Gaussian energy resolution function, followed by several additional steps, to obtain jets. A detailed description of the detector simulation and reconstruction with ATLFAST is given in [11]. For the minimum transverse energy threshold for clusters and for the minimum transverse momentum threshold for isolated photons the default values in ATHENA 10.0.1 were used which are both $5 \mathrm{GeV}$. These values differ from the ones in [11].

\subsection{Signal Events}

Signal events for the weak boson fusion process were generated with the program vbfnlo [13] which contains the anomalous Higgs boson couplings described in the previous section. In the version of vbfnlo used in this study, unweighted events can only be generated at leading order, hence the program was used at leading order. The version of vbfnlo used in this study does not assign spins and masses to the final-state $\tau$ leptons. They were added by modifying the event data files, following a prescription provided by the author of the program [14].

\footnotetext{
${ }^{1} \Delta R=\sqrt{\Delta \eta^{2}+\Delta \phi^{2}}$ is a measure of the distance in the $\eta$ - $\phi$-plane.
} 
When generating events with vbfnlo one has to take care that the "scale of the event", which is stored in the Les Houches variable SCALUP, is set to a suitable value. If the scale of the event is not set, indicated by a value of the variable SCALUP of -1 , Pythia uses the centre-of-mass energy of the parton-parton system $\hat{s}$ which is generally considered too large in weak boson fusion. In this study, the factorisation and renormalisation scales in vbfnlo were set for each quark line to the momentum transfer of the corresponding $W$ or $Z$ boson. In the Les Houches file, the variable SCALUP was set to the $W$ boson mass for $W W$ fusion events and to the $Z$ boson mass for $Z Z$ fusion events.

When using parton-level events generated by an external generator, the value of SCALUP is multiplied in Pythia by the parameter PARP(67) to determine the cut-off scale for the initial-state parton shower and by the parameter PARP(71) to determine the cut-off scale for the final-state parton shower. These multiplicative factors are not applied when the weak boson fusion events are generated by Pythia itself. To achieve consistency between the results from events generated with vbfnlo when used with standard model couplings and those from events generated with Pythia, the parameter PARP $(71)$ was changed from the default value of 4 in ATHENA 10.0.1 to 1 while for the parameter PARP(67) the default value of 1 was kept. The results from Pythia are compared to the results from vbfnlo with standard model couplings in section 4.6 after the discussion of the event selection.

Signal events were generated for the Higgs boson decay channels $H \rightarrow \tau^{+} \tau^{-} \rightarrow l l+4 \nu$ and $H \rightarrow \tau^{+} \tau^{-} \rightarrow l h+3 \nu$ at a Higgs boson mass of $120 \mathrm{GeV}$ and for the Higgs boson decay channel $H \rightarrow W^{+} W^{-} \rightarrow l l \nu \nu$ at a Higgs boson mass of $160 \mathrm{GeV}$. In the channel $H \rightarrow \tau^{+} \tau^{-} \rightarrow l l+4 \nu$ only leptonic decay modes were allowed for the decays of both $\tau$ leptons while in the channel $H \rightarrow \tau^{+} \tau^{-} \rightarrow l h+3 \nu$ only leptonic decay modes were allowed for the decay of one $\tau$ lepton and only hadronic decay modes, with all possible multiplicities of charged hadrons, were allowed for the decay of the other $\tau$ lepton. According to the analyses at leading order of the discovery potential for a standard model Higgs boson with the ATLAS detector (see figure 2) those are the channels with the highest expected signal significances at the respective Higgs boson masses. The mass values are chosen to lie within the region which has not yet been experimentally excluded for a standard model Higgs boson.

For each channel 4.5 million events were generated with standard model (SM) couplings. In addition, for each channel 1.5 million events with purely $\mathrm{CP}$ even anomalous couplings $\left(g_{5 e}^{H Z Z}=g_{5 e}^{H W W} / \cos ^{2} \theta_{w}=1\right.$, "CPE" $)$ and 1.5 million events with purely CP odd anomalous couplings $\left(g_{5 o}^{H Z Z}=g_{5 o}^{H W W} / \cos ^{2} \theta_{w}=1\right.$, "CPO") were generated. In these samples the standard model couplings were set to 0 . The cross sections for the standard model case were taken from [1]. The cross sections for the samples with purely anomalous couplings depend directly on the undetermined strengths of the anomalous couplings. Since these samples are used in a hypothesis test in which only the shape of a distribution is tested, no a priori assumption about the total cross sections was made.

\subsection{Background Events}

- Events in which the Higgs boson is produced by gluon-gluon fusion were generated for each signal channel with Pythia. This process is listed as a background process here because the focus of this study is not the discovery potential for a Higgs boson but rather the determination of the couplings of the Higgs boson to weak bosons, to which gluon-gluon fusion does not contribute. This labelling has no effect on the treatment of this process in the analysis. For the calculation of the expected signal significance in section 4 the process is nevertheless treated as part of the signal to facilitate comparison with studies of the discovery potential where this convention is generally used. 


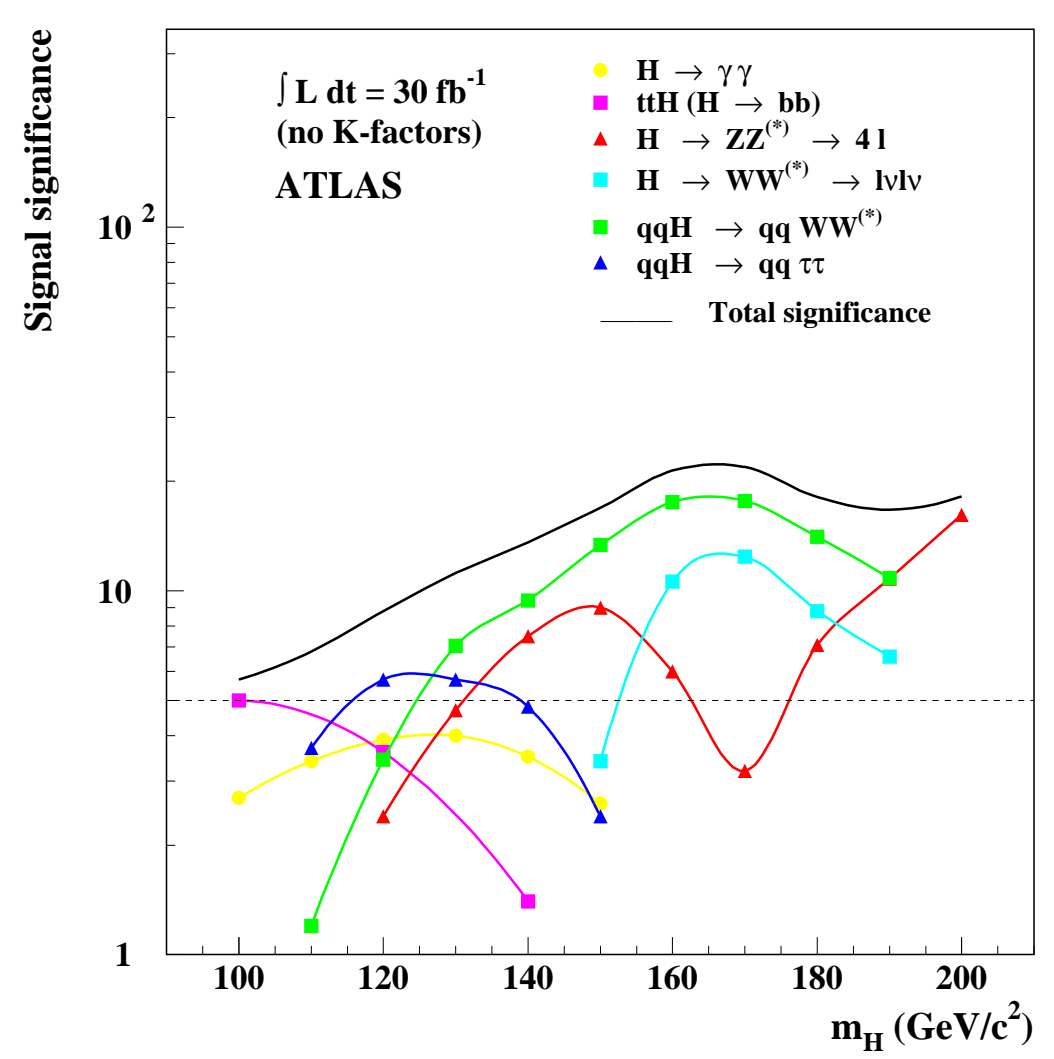

Figure 2: Expected signal significance with the ATLAS experiment for a standard model Higgs boson in the region of small to intermediate Higgs boson masses [1].

- $t \bar{t}$ events were generated with Pythia. In this event sample $W$ bosons were forced to decay leptonically.

- Wt events were generated with TopReX 4.06 [15]. All decay channels were allowed. A filter was applied to the generated events that required at least one lepton with $|\eta|<3$ and $p_{T}>8 \mathrm{GeV}$.

- WWjj events from electroweak production were generated with MadEvent [16]. The decays of the $W$ bosons were calculated with Pythia. Only leptonic decay modes were allowed. For consistency with the signal event generation the parameter PARP(71) in Pythia was set to 1.

- WWjj events from QCD production were generated with MadEvent [16]. Again, for the $W$ bosons only leptonic decay modes were allowed. Because of technical problems with Pythia in ATHENA 10.0.1 the decays were calculated with MadEvent for this process. Since this is a QCD process the parameter PARP(71) was left at its default value.

- Zjj events from electroweak and from QCD production were generated with MadCUP which is based on calculations presented in $[17,18]$ and references therein. Leptonic decays of the $Z$ boson were calculated by MadCUP.

An overview of the cuts applied at the generator level is given in table 4. A list of all generated Monte Carlo samples is given in table 5. 


\begin{tabular}{|c|c|c|c|c|c|}
\hline & WBF & WWjj(EW) & WWjj(QCD) & Zjj(EW) & Zjj(QCD) \\
\hline all final state quarks: & & & & & \\
$p_{T}$ & $>5 \mathrm{GeV}$ & $>15 \mathrm{GeV}$ & $>15 \mathrm{GeV}$ & $>5 \mathrm{GeV}$ & $>20 \mathrm{GeV}$ \\
$E$ & $>5 \mathrm{GeV}$ & - & - & - & - \\
$|\eta|$ & - & $<5.5$ & $<6$ & - & $<6$ \\
$\Delta R$ & - & $>0.3$ & - & - & - \\
$|\Delta \eta|$ & - & - & - & - & $>3$ \\
$m_{q q}$ & - & - & $>200 \mathrm{GeV}$ & - & $>400 \mathrm{GeV}$ \\
\hline $\begin{array}{c}\text { all final state leptons: } \\
p_{T}\end{array}$ & - & - & - & $>5 \mathrm{GeV}$ & $>5 \mathrm{GeV}$ \\
$|\eta|$ & - & - & - & - & $<3$ \\
$m_{l l}$ & - & - & - & $(76.19-251.19)$ & $(75-160)$ \\
& & - & - & - & $\mathrm{GeV}$ \\
\hline$R_{q l}$ & - & - & - & & $>0.3$ \\
\hline
\end{tabular}

Table 4: Cuts applied at the generator level (i.e. at parton level). For Monte Carlo events generated with Pythia and TopReX the standard settings of Pythia and TopReX in ATHENA 10.0.1 were used.

\section{Event Selection}

Events are selected following the cut analyses described in [1] which are optimised to give the maximum signal significance. In the following some selection criteria are motivated and lists of the cuts are given. Details about the cut analyses can be found in [1] and references therein. Some cuts are modified in the channel $H \rightarrow \tau^{+} \tau^{-} \rightarrow l l+4 \nu$ where the cut on the azimuthal angle between the tagging jets is omitted since this is the sensitive observable that is used later in the analysis. The changes are indicated in the list of cuts.

\subsection{Motivation of the Selection Criteria}

A typical feature of WBF events is the occurrence of two so-called tagging jets with a large pseudorapidity gap between them. They emerge from the two quarks that take part in the hard process and that are scattered preferentially in the forward direction. The tagging jets are required to have a minimum $p_{T}$ and a minimum distance in $\eta$ from each other. Since in contrast to QCD processes there is no colour flow between the quarks, there are usually no jets with a large $p_{T}$ in the region between the tagging jets which is utilised by applying a veto on additional central jets.

In the channel $H \rightarrow W^{+} W^{-} \rightarrow l l \nu \nu$ the directions of the final state leptons are correlated. Since the Higgs boson is scalar the spins of the $W$ bosons are aligned opposite to each other and the lepton and anti-lepton are emitted preferentially in the same direction. This is used by cutting on the angular variables of the leptons.

The fact that the transverse momentum of the tagging jets should be balanced by the transverse momentum of the Higgs boson is used in $H \rightarrow W^{+} W^{-} \rightarrow l l \nu \nu$ by cutting on the absolute value of the vector

$$
p_{T}^{t o t}=p_{T}^{l, 1}+p_{T}^{l, 2}+p_{T}^{m i s s}+p_{T}^{j, 1}+p_{T}^{j, 2}
$$

with the transverse momenta of the tagging jets $p_{T}^{j, 1}$ and $p_{T}^{j, 2}$, the transverse momenta of the leptons $p_{T}^{l, 1}$ and $p_{T}^{l, 2}$ and the missing transverse momentum $p_{T}^{m i s s}$ which is at parton level 


\begin{tabular}{|c|c|c|c|}
\hline process & generator & cross section & number of events \\
\hline $\begin{array}{l}\text { WBF: } H \rightarrow \tau^{+} \tau^{-} \rightarrow l l+4 \nu \\
m_{H}=120 \mathrm{GeV}, \mathrm{SM} \text { couplings }\end{array}$ & vbfnlo & $37.67 \mathrm{fb}$ & 4500000 \\
\hline $\begin{array}{l}\text { WBF }: H \rightarrow \tau^{+} \tau^{-} \rightarrow l l+4 \nu \\
m_{H}=120 \mathrm{GeV}, \mathrm{CPE} \text { couplings }\end{array}$ & vbfnlo & - & 1500000 \\
\hline $\begin{array}{l}\text { WBF }: H \rightarrow \tau^{+} \tau^{-} \rightarrow l l+4 \nu \\
m_{H}=120 \mathrm{GeV}, \mathrm{CPO} \text { couplings }\end{array}$ & vbfnlo & - & 1500000 \\
\hline $\begin{array}{l}\text { WBF: } H \rightarrow \tau^{+} \tau^{-} \rightarrow l \nu \nu+\text { hadron } \\
m_{H}=120 \mathrm{GeV}, \mathrm{SM} \text { couplings }\end{array}$ & vbfnlo & $138.7 \mathrm{fb}$ & 4500000 \\
\hline $\begin{array}{l}\text { WBF: } H \rightarrow \tau^{+} \tau^{-} \rightarrow l \nu \nu+\text { hadron } \\
m_{H}=120 \mathrm{GeV}, \mathrm{CPE} \text { couplings }\end{array}$ & vbfnlo & - & 1500000 \\
\hline $\begin{array}{l}\text { WBF: } H \rightarrow \tau^{+} \tau^{-} \rightarrow l \nu \nu+\text { hadron } \\
m_{H}=120 \mathrm{GeV}, \mathrm{CPO} \text { couplings }\end{array}$ & vbfnlo & - & 1500000 \\
\hline $\begin{array}{l}\text { WBF: } H \rightarrow W^{+} W^{-} \rightarrow l l \nu \nu \\
m_{H}=160 \mathrm{GeV}, \mathrm{SM} \text { couplings }\end{array}$ & vbfnlo & $303.3 \mathrm{fb}$ & 4500000 \\
\hline $\begin{array}{l}\text { WBF: } H \rightarrow W^{+} W^{-} \rightarrow l l \nu \nu \\
m_{H}=160 \mathrm{GeV}, \mathrm{CPE} \text { couplings }\end{array}$ & vbfnlo & - & 1500000 \\
\hline $\begin{array}{l}\text { WBF }: H \rightarrow W^{+} W^{-} \rightarrow l l \nu \nu \\
m_{H}=160 \mathrm{GeV}, \mathrm{CPO} \text { couplings }\end{array}$ & vbfnlo & - & 1500000 \\
\hline $\begin{array}{l}\text { gg fusion: } H \rightarrow \tau^{+} \tau^{-} \rightarrow l l+4 \nu \\
m_{H}=120 \mathrm{GeV}\end{array}$ & Pythia & $170.1 \mathrm{fb}$ & 300000 \\
\hline $\begin{array}{l}\text { gg fusion: } H \rightarrow \tau^{+} \tau^{-} \rightarrow l h+3 \nu \\
m_{H}=120 \mathrm{GeV}\end{array}$ & Pythia & $313.2 \mathrm{fb}$ & 900000 \\
\hline $\begin{array}{l}\text { gg fusion: } H \rightarrow W^{+} W^{-} \rightarrow l l \nu \nu \\
m_{H}=160 \mathrm{GeV}\end{array}$ & Pythia & $991.2 \mathrm{fb}$ & 1200000 \\
\hline $\mathrm{t} \overline{\mathrm{t}}, W$ decays leptonic & Pythia & $51.66 \mathrm{pb}$ & 65000000 \\
\hline Wt, one lepton filter (see text) & TopReX & $26.66 \mathrm{pb}$ & 34473508 \\
\hline WWjj(EW), $W$ decays leptonic & MadEvent & $95.4 \pm 1.1 \mathrm{fb}$ & 148000 \\
\hline WWjj(QCD), $W$ decays leptonic & MadEvent & $1.392 \pm 0.004 \mathrm{pb}$ & 1911562 \\
\hline Zjj(EW), $Z$ decays leptonic & MadCUP & $2.764 \mathrm{pb}$ & 3152807 \\
\hline Zjj(QCD), $Z$ decays leptonic & MadCUP & $26.12 \mathrm{pb}$ & 27951817 \\
\hline
\end{tabular}

Table 5: List of the generated Monte Carlo event samples. The statistical errors on the cross sections are known and non-negligible only for the processes WWjj(EW) and WWjj(QCD). The cross sections of the signal processes and the branching ratios for the Higgs boson decays were taken from [1]. Branching ratios for the $W$ boson decays were taken from [19]. The cross sections that are given for background processes are those calculated by the respective programs with the cuts at generator level as given in table 4 . For the case of purely anomalous couplings no cross sections were calculated. 
given by the sum of the transverse momenta of the neutrinos. Since the absolute value of $p_{T}^{\text {tot }}$ will only be small as long as there is no additional hard radiation this cut is correlated with the jet veto.

The contribution of the background processes $Z \rightarrow e^{+} e^{-} / \mu^{+} \mu^{-}$in the channel $H \rightarrow$ $W^{+} W^{-} \rightarrow l l \nu \nu$ can be suppressed by requiring the invariant mass of same flavour lepton pairs to lie outside a window around the $Z$ boson mass. Additional suppression can be achieved by a cut on the transverse mass of the lepton-neutrino system

$$
m_{T}\left(l l p_{T}^{m i s s}\right)=\sqrt{2 P_{T}(l l) P_{T}^{m i s s}(1-\cos \Delta \phi)}
$$

with the angle $\Delta \phi$ between the sum vector of the transverse momenta of the leptons $P_{T}(l l)$ and the missing transverse momentum vector.

In the $H \rightarrow \tau^{+} \tau^{-}$channels the Higgs boson mass can be reconstructed from the transverse momenta of the decay products of the $\tau$ leptons and the missing transverse momentum. This reconstruction is described in section 4.2. By selecting a window around the Higgs boson mass the $Z \rightarrow \tau^{+} \tau^{-}$background can be suppressed.

Contributions from background processes with top quarks can be suppressed by requiring that the tagging jets have not been tagged as $b$-jets. For the tagging of b-jets the default efficiency in ATHENA 10.0.1 of $50 \%$ is used. The probability for c-jets to be mistagged as b-jets is taken as $1 / 10.9$, the mistag probability for the remaining jets is $1 / 231$.

A lepton reconstruction efficiency of $90 \%$ is applied [20].

\subsection{Reconstruction of the Higgs Boson Mass in $H \rightarrow \tau^{+} \tau^{-}$}

The mass of the boson in the decay $H / Z \rightarrow \tau^{+} \tau^{-} \rightarrow l l+4 \nu$ can be reconstructed in the approximation that the transverse momenta of all decay products of a $\tau$ lepton are parallel to the transverse momentum of the $\tau$ lepton, the so-called collinear approximation. This approximation is justified as long as the absolute values of the transverse momenta of the $\tau$ leptons in the laboratory frame are sufficiently large. This is usually the case due to the large mass difference between the decaying boson and the $\tau$ lepton and the transverse momentum of the boson.

The mass reconstruction method can be applied both in the $H / Z \rightarrow \tau^{+} \tau^{-} \rightarrow l l+4 \nu$ and the $H / Z \rightarrow \tau^{+} \tau^{-} \rightarrow l h+3 \nu$ channels. The following refers to the case of the decay $\tau^{+} \tau^{-} \rightarrow l l+4 \nu$. For the case of the decay $\tau^{+} \tau^{-} \rightarrow l h+3 \nu$ one of the lepton momenta has to be replaced by the corresponding momentum of the object identified as a $\tau$ jet.

In the following all leptons are treated as massless. The fractions $x_{\tau_{1}}$ and $x_{\tau_{2}}$ of the $\tau$ lepton momenta that are carried by the observed leptons are defined as

$$
x_{\tau_{i}} p_{\tau_{i}}=p_{l_{i}} .
$$

The fractions $x_{\tau_{1}}$ and $x_{\tau_{2}}$ can be calculated by using momentum conservation in the transverse plane:

$$
p_{\tau_{1}}^{T}+p_{\tau_{2}}^{T}=\frac{p_{l_{1}}^{T}}{x_{\tau_{1}}}+\frac{p_{l_{2}}^{T}}{x_{\tau_{2}}}=p_{l_{1}}^{T}+p_{l_{2}}^{T}+p_{m i s s}^{T}
$$

Solving for $x_{\tau_{1}}$ and $x_{\tau_{2}}$ gives:

$$
\begin{aligned}
x_{\tau_{1}} & =\frac{p_{l_{2}}^{x} p_{l_{1}}^{y}-p_{l_{1}}^{x} p_{l_{2}}^{y}}{p_{l_{2}}^{x} p_{l_{1}}^{y}+p_{l_{2}} p_{m i s s}^{y}-p_{l_{2}}^{y} p_{l_{1}}^{x}-p_{l_{2}}^{y} p_{m i s s}^{x}} \\
x_{\tau_{2}} & =\frac{p_{l_{1}}^{x} p_{l_{2}}^{y}-p_{l_{2}}^{x} p_{l_{1}}^{y}}{p_{l_{1}}^{x} p_{l_{2}}^{y}+p_{l_{1}}^{x} p_{m i s s}^{y}-p_{l_{1}}^{y} p_{l_{2}}^{x}-p_{l_{1}}^{y} p_{m i s s}^{x}}
\end{aligned}
$$


From this the mass of the boson is calculated as

$$
m_{\tau \tau}=\sqrt{\left(p_{\tau_{1}}+p_{\tau_{2}}\right)^{2}}=\sqrt{2 p_{\mu \tau_{1}} p_{\tau_{2}}^{\mu}}=\frac{m_{l l}}{\sqrt{x_{\tau_{1}} x_{\tau_{2}}}} .
$$

\subsection{Selection Cuts for $H \rightarrow W^{+} W^{-} \rightarrow l l \nu \nu$}

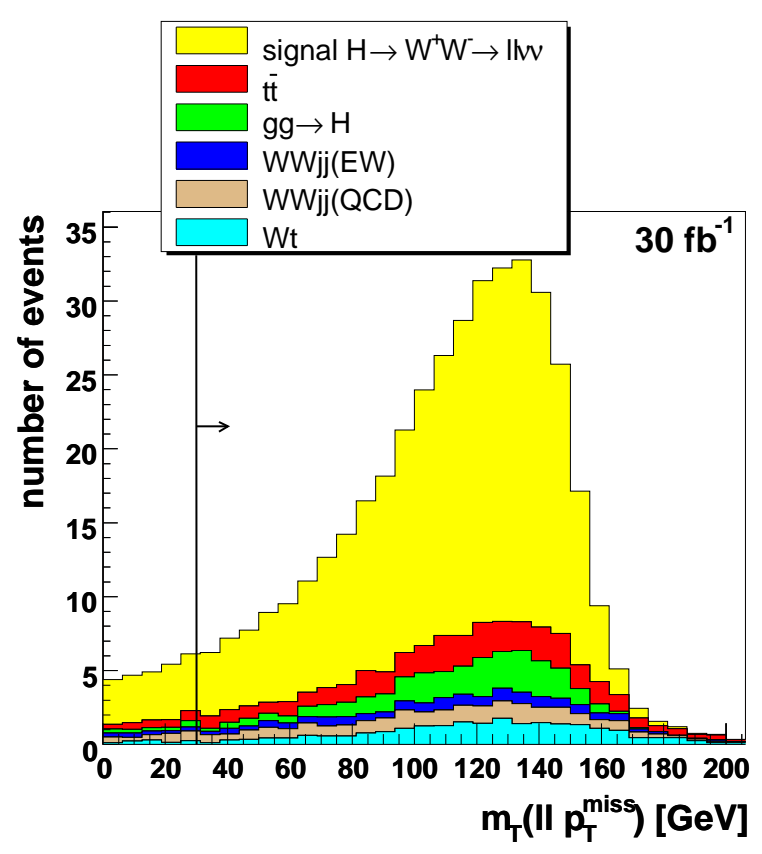

Figure 3: Distribution of the expected number of events in the last cut variable $m_{T}\left(l l p_{T}^{m i s s}\right)$ in the channel $H \rightarrow W^{+} W^{-} \rightarrow l l \nu \nu$ for an integrated luminosity of $30 \mathrm{fb}^{-1}$. All cuts except the last one have been applied. All generated Monte Carlo events have been used and the distributions have been scaled to the respective expected number of events.

1. jet and lepton acceptance: Two observed candidates for the tagging jets are required. Tagging jet candidates are the jet with the largest $p_{T}$ in the region $\eta<0$ and the jet with the largest $p_{T}$ in the region $\eta>0$. In addition two observed leptons with $|\eta|<2.5$ are required. One lepton must have $p_{T}>20 \mathrm{GeV}$ and the other one $p_{T}>15 \mathrm{GeV}$.

2. b-jet veto: The tagging jet candidates must not have been tagged as $b$-jets.

3. tagging jet identification: One tagging jet candidate must have $p_{T}>40 \mathrm{GeV}$, the other one must have $p_{T}>20 \mathrm{GeV}$. There must be a gap in pseudorapidity of at least $\Delta \eta>3.8$ between the tagging jet candidates. The leptons must have pseudorapidities between those of the tagging jet candidates: $\eta_{t a g}^{\text {min }}<\eta_{l_{1,2}}<\eta_{t a g}^{\max }$.

4. lepton cuts: For the decay leptons $\Delta \phi_{l l}<1.05, \Delta R_{l l}<1.8, \cos \theta_{l l}>0.2, m_{l l}<85 \mathrm{GeV}$ and $p_{T}(l)<120 \mathrm{GeV}$ are required, with the azimuthal angle around the beam axis between the lepton momenta $\Delta \phi_{l l}$, the distance in the $\eta$ - $\phi$-plane between the lepton momenta $\Delta R_{l l}$, the cosine of the polar angle with respect to the beam axis between the lepton momenta $\cos \theta_{l l}$, the invariant mass of the lepton pair $m_{l l}$ and the transverse momentum of each lepton $p_{T}(l)$. 


\begin{tabular}{|l|c|c|c|}
\hline & $\begin{array}{c}\sigma \cdot \mathrm{BR} \\
\text { before cuts }\end{array}$ & $\begin{array}{c}\sigma \cdot \mathrm{BR} \\
\text { after cuts }\end{array}$ & $\begin{array}{c}\text { events expected for } 30 \mathrm{fb}^{-1} \\
\text { after cuts }\end{array}$ \\
\hline signal & $303.3 \mathrm{fb}$ & $9.46 \pm 0.02 \mathrm{fb}$ & $284 \pm 1$ \\
gg fusion & $991.2 \mathrm{fb}$ & $0.96 \pm 0.03 \mathrm{fb}$ & $29 \pm 1$ \\
t $\mathrm{t}$ & $51.66 \mathrm{pb}$ & $1.23 \pm 0.03 \mathrm{fb}$ & $37 \pm 1$ \\
Wt & $26.66 \mathrm{pb}$ & $0.77 \pm 0.02 \mathrm{fb}$ & $23 \pm 1$ \\
WWjj(EW) & $95.4 \pm 1.1 \mathrm{fb}$ & $0.43 \pm 0.02 \mathrm{fb}$ & $13 \pm 1$ \\
WWjj(QCD) & $1.392 \pm 0.004 \mathrm{pb}$ & $0.69 \pm 0.02 \mathrm{fb}$ & $21 \pm 1$ \\
\hline
\end{tabular}

Table 6: Results of the event selection for the channel $H \rightarrow W^{+} W^{-} \rightarrow l l \nu \nu$. Statistical errors are given where known and non-negligible.

5. $Z \rightarrow \tau^{+} \tau^{-}$suppression: Events with $x_{\tau_{1}}>0$ and $x_{\tau_{2}}>0$ and $\left|m_{\tau \tau}-m_{Z}\right|<25 \mathrm{GeV}$ are discarded.

6. invariant mass of the tagging jet pair: $m_{j j}>550 \mathrm{GeV}$.

7. momentum balance: $\left|p_{T}^{t o t}\right|<30 \mathrm{GeV}$.

8. central jet veto: No jets with $p_{T}>20 \mathrm{GeV}$ that are not tagging jets are allowed in the region $|\eta|<3.2$.

9. $Z \rightarrow e^{+} e^{-} / \mu^{+} \mu^{-}$suppression: Events with same flavour lepton pairs are required to have $m_{l l}<75 \mathrm{GeV}$ and $p_{T}^{\text {miss }}>30 \mathrm{GeV}$.

10. $m_{T}\left(l l p_{T}^{m i s s}\right)$ cut: $m_{T}\left(l l p_{T}^{m i s s}\right)>30 \mathrm{GeV}$.

The results of the cut analysis in this channel are listed in table 6. The distribution in the last cut variable is shown in figure 3. Due to the choice of cuts at the generator level the Zjj event samples could not be used as background samples in this channel. However, it was shown in [1] that the contribution from $\mathrm{Zjj}$ is small after all cuts. The expected signal significance, using Poisson statistics and treating the gluon-gluon fusion process as a part of the signal, is 24 for an integrated luminosity of $30 \mathrm{fb}^{-1}$ and a Higgs boson mass of $160 \mathrm{GeV}$.

\subsection{Selection Cuts for $H \rightarrow \tau^{+} \tau^{-} \rightarrow l l+4 \nu$}

1. jet and lepton acceptance: Two observed candidates for the tagging jets are required. The candidates are selected as in the channel $H \rightarrow W^{+} W^{-} \rightarrow l l \nu \nu$. In addition, cuts based on the current trigger conditions for leptons (one- or two-lepton trigger) have to be satisfied. These cuts require either one isolated electron with $p_{T}>25 \mathrm{GeV}$ or a muon with $p_{T}>20 \mathrm{GeV}$ or two isolated electrons with $p_{T}>15 \mathrm{GeV}$ or two muons with $p_{T}>10 \mathrm{GeV}$ or an isolated electron with $p_{T}>15 \mathrm{GeV}$ together with a muon with $p_{T}>10 \mathrm{GeV}$. At least two observed leptons with $|\eta|<2.5$ are required. The two leptons with the largest $p_{T}$ are selected.

2. b-jet veto: The tagging jet candidates must not have been tagged as $b$-jets.

3. tagging jet identification: One tagging jet candidate must have $p_{T}>50 \mathrm{GeV}$, the other one must have $p_{T}>30 \mathrm{GeV}$. There must be a gap in pseudorapidity of $\Delta \eta>4.4$ between the tagging jet candidates. The leptons must have pseudorapidities between those of the tagging jet candidates. There must be a gap in pseudorapidity of $\Delta \eta>0.7$ 


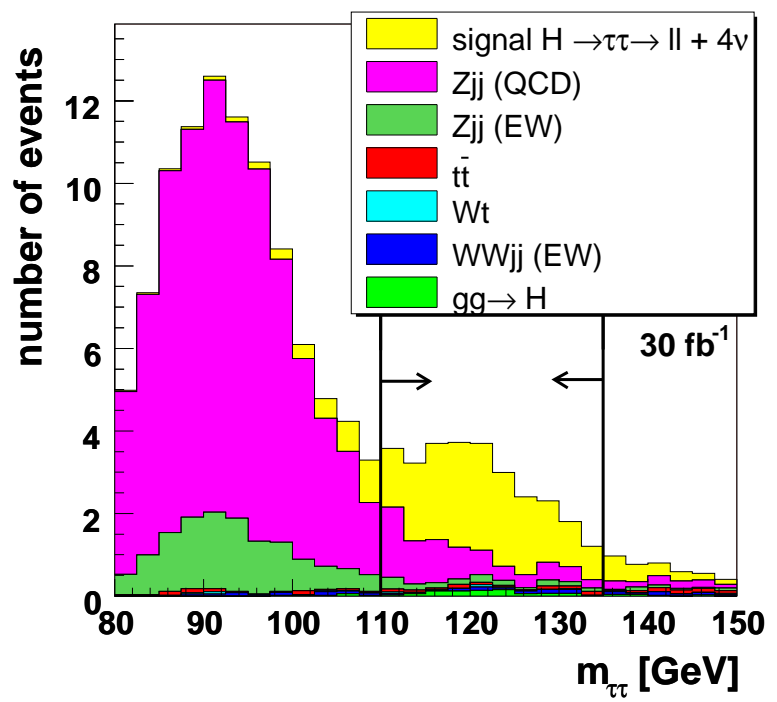

Figure 4: Distribution of the expected number of events in the last cut variable, the invariant mass of the $\tau$ lepton pair, in the channel $H \rightarrow \tau^{+} \tau^{-} \rightarrow l l+4 \nu$ for an integrated luminosity of $30 \mathrm{fb}^{-1}$. All cuts except the last one have been applied. All generated Monte Carlo events have been used and the distributions have been scaled to the respective expected number of events.

between a tagging jet candidate and a lepton: $\eta_{t a g}^{\min }+0.7<\eta_{l_{1,2}}<\eta_{t a g}^{\max }-0.7$. The requirement of a gap in $\eta$ between the momenta of the leptons and the tagging jets was added with respect to [1].

4. missing transverse momentum: $p_{T}^{\text {miss }}>50 \mathrm{GeV}$.

5. invariant mass of the tagging jet pair: $m_{j j}>700 \mathrm{GeV}$.

6. central jet veto: No jets with $p_{T}>20 \mathrm{GeV}$ that are not tagging jets are allowed in the region $|\eta|<3.2$.

7. distance between lepton momenta: $\Delta R_{l l}<2.4$ is required. This cut was tightened with respect to [13] where $\Delta R_{l l}<2.6$ was required.

8. reconstruction of $\tau$ lepton momenta: $x_{\tau_{1}}>0$ and $x_{\tau_{2}}>0$ and $x_{\tau_{1}}^{2}+x_{\tau_{2}}^{2}<1$ are required.

9. Higgs boson mass window: $110 \mathrm{GeV}<m_{\tau \tau}<135 \mathrm{GeV}$.

The results of the cut analysis in this channel are listed in table 7. The distribution in the last cut variable is shown in figure 4. The expected signal significance, using Poisson statistics and treating the gluon-gluon fusion process as a part of the signal, is 4.7 for an integrated luminosity of $30 \mathrm{fb}^{-1}$ and a Higgs boson mass of $120 \mathrm{GeV}$.

\subsection{Selection Cuts for $H \rightarrow \tau^{+} \tau^{-} \rightarrow l h+3 \nu$}

1. jet and lepton acceptance: Two observed candidates for the tagging jets are required. The candidates are selected as in the channel $H \rightarrow W^{+} W^{-} \rightarrow l l \nu \nu$. In addition, one observed isolated electron with $|\eta|<2.5$ and $p_{T}>25 \mathrm{GeV}$ or one observed muon with $|\eta|<2.5$ and $p_{T}>20 \mathrm{GeV}$ is required (single-lepton trigger requirement). 


\begin{tabular}{|l|c|c|c|}
\hline & $\begin{array}{c}\sigma \cdot \mathrm{BR} \\
\text { before cuts }\end{array}$ & $\begin{array}{c}\sigma \cdot \mathrm{BR} \\
\text { after cuts }\end{array}$ & $\begin{array}{c}\text { events expected for } 30 \mathrm{fb}^{-1} \\
\text { after cuts }\end{array}$ \\
\hline signal & $37.67 \mathrm{fb}$ & $0.61 \mathrm{fb}$ & 18 \\
gg fusion & $170.1 \mathrm{fb}$ & $0.03 \mathrm{fb}$ & 1 \\
$\mathrm{t} \overline{\mathrm{t}}$ & $51.66 \mathrm{pb}$ & $0.02 \mathrm{fb}$ & 1 \\
$\mathrm{Wt}$ & $26.67 \mathrm{pb}$ & $0.01 \mathrm{fb}$ & 0 \\
WWjj$(\mathrm{EW})$ & $95.4 \pm 1.1 \mathrm{fb}$ & $0.02 \mathrm{fb}$ & 1 \\
Zjj(EW) & $2.76 \mathrm{pb}$ & $0.05 \pm 0.01 \mathrm{fb}$ & 2 \\
Zjj(QCD) & $26.12 \mathrm{pb}$ & $0.23 \pm 0.01 \mathrm{fb}$ & 7 \\
\hline
\end{tabular}

Table 7: Results of the event selection for the channel $H \rightarrow \tau^{+} \tau^{-} \rightarrow l l+4 \nu$. Statistical errors are given where known and non-negligible.

2. b-jet veto: The tagging jet candidates must not have been tagged as $b$-jets.

3. hadronic $\tau$ lepton decay: One jet labelled as a $\tau$-jet as described in [11] is required. The jet must have $p_{T}>40 \mathrm{GeV}$.

4. tagging jet identification: One tagging jet candidate must have $p_{T}>40 \mathrm{GeV}$, the other one must have $p_{T}>20 \mathrm{GeV}$. There must be a gap in pseudorapidity of $\Delta \eta>4.4$ between the tagging jet candidates. The leptons must have pseudorapidities between those of the tagging jet candidates: $\eta_{t a g}^{\min }<\eta_{l, h}<\eta_{t a g}^{\max }$.

5. reconstruction of $\tau$ lepton momenta: $0<x_{\tau_{l}}<0.75$ and $0<x_{\tau_{h}}<1$ are required.

6. transverse mass: $m_{T}\left(l p_{T}^{\text {miss }}\right)=\sqrt{2 p_{T}(l) p_{T}^{\text {miss }}(1-\cos \Delta \phi)}<30 \mathrm{GeV}$.

7. missing transverse momentum: $p_{T}^{\text {miss }}>30 \mathrm{GeV}$.

8. invariant mass of the tagging jet pair: $m_{j j}>700 \mathrm{GeV}$.

9. central jet veto: No jets with $p_{T}>20 \mathrm{GeV}$ are allowed in the pseudorapidity region between the tagging jets.

10. Higgs boson mass window: $110 \mathrm{GeV}<m_{\tau \tau}<135 \mathrm{GeV}$.

The results of the cut analysis in this channel are listed in table 8 . The distribution in the last cut variable is shown in figure 5 . The analysis in this channel requires the identification of the decay products coming from hadronic $\tau$ lepton decays. Since the identification of hadronic $\tau$ lepton decays in ATLFAST-B does not give correct results in ATHENA 10.0.1 [21] the identification efficiency is applied by discarding $50 \%$ of all events. It is assumed that the main background in this channel comes from hadronic $\tau$ lepton decays in the process $Z \rightarrow \tau^{+} \tau^{-}$. The possibility of misidentified hadronic $\tau$ decay products is not taken into account. Hence, only the Zjj and gluon-gluon fusion background samples are used for the analysis in this channel. Contributions from other background processes were evaluated in [1] and shown to be small after all cuts. Since the energy calibration in ATLFAST-B does not give the correct results for hadronic $\tau$ decay products [21] the uncalibrated energy is used for the jets labelled as $\tau$-jets. The expected signal significance, using Poisson statistics and treating the gluon-gluon fusion process as a part of the signal, is $4.3 \mathrm{for}$ an integrated luminosity of $30 \mathrm{fb}^{-1}$ and a Higgs boson mass of $120 \mathrm{GeV}$. 


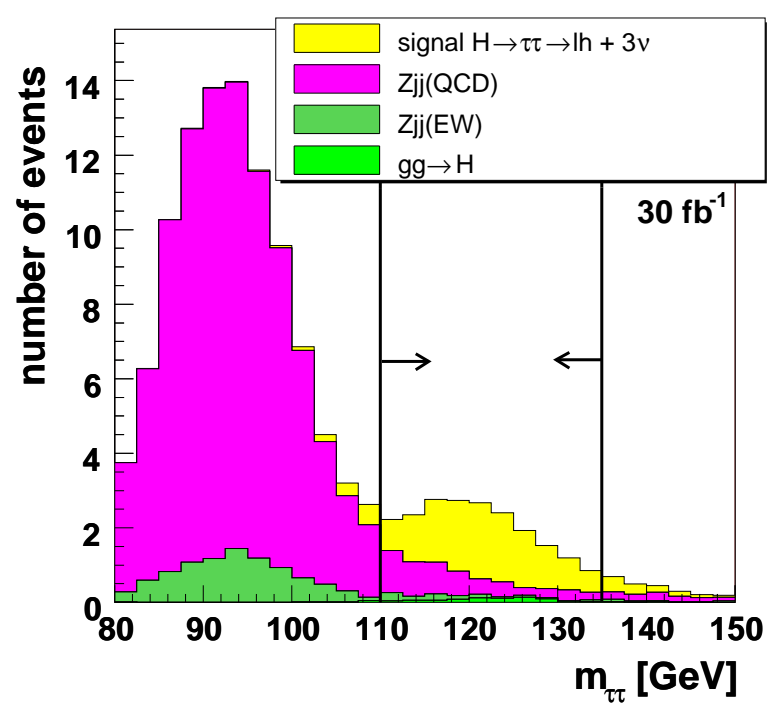

Figure 5: Distribution of the expected number of events in the last cut variable, the invariant mass of the $\tau$ lepton pair, in the channel $H \rightarrow \tau^{+} \tau^{-} \rightarrow l h+3 \nu$ for an integrated luminosity of $30 \mathrm{fb}^{-1}$. All cuts except the last one have been applied. All generated Monte Carlo events have been used and the distributions have been scaled to the respective expected number of events.

\begin{tabular}{|l|c|c|c|}
\hline & $\begin{array}{c}\sigma \cdot \mathrm{BR} \\
\text { before cuts }\end{array}$ & $\begin{array}{c}\sigma \cdot \mathrm{BR} \\
\text { after cuts }\end{array}$ & $\begin{array}{c}\text { events expected for } 30 \mathrm{fb}^{-1} \\
\text { after cuts }\end{array}$ \\
\hline signal & $138.7 \mathrm{fb}$ & $0.46 \mathrm{fb}$ & 14 \\
gg fusion & $313.2 \mathrm{fb}$ & $0.02 \mathrm{fb}$ & 1 \\
Zjj(EW) & $2.76 \mathrm{pb}$ & $0.03 \pm 0.01 \mathrm{fb}$ & 1 \\
Zjj(QCD) & $26.12 \mathrm{pb}$ & $0.18 \pm 0.01 \mathrm{fb}$ & 5 \\
\hline
\end{tabular}

Table 8: Results of the event selection for the channel $H \rightarrow \tau^{+} \tau^{-} \rightarrow l h+3 \nu$. Statistical errors are given where known and non-negligible.

\subsection{Comparison of vbfnlo with Pythia}

The event samples generated with vbfnlo with standard model couplings are compared to WBF event samples generated with Pythia to check the consistency between the two generators. It was already pointed out in section 3.1 that the parameter $\operatorname{PARP}(71)$, which controls the cut-off scale for the final state parton shower, has to be changed from its default value in ATHENA 10.0.1 of 4 to 1 when vbfnlo is used as an external event generator to achieve the same parton shower behaviour as when Pythia is used to generate the events. A variable that is particularly sensitive to the value of $\operatorname{PARP}(71)$ is $\left|p_{T}^{\text {tot }}\right|$. The distribution of $\left|p_{T}^{\text {tot }}\right|$ is shown in figure 6 for events generated with vbfnlo for two different values of PARP(71) and for events generated with Pythia. One can see that the distributions from vbfnlo and Pythia agree for $\operatorname{PARP}(71)=1$ while they differ significantly for $\operatorname{PARP}(71)=4$. The efficiency of the cut on $\left|p_{T}^{\text {tot }}\right|$ increases by about $15 \%$ relative to the value for $\operatorname{PARP}(71)=4$ when PARP $(71)$ is changed from 4 to 1 . The efficiency of the whole event selection increases by about $30 \%$. There is no a priori reason to prefer one value of PARP $(71)$ over the other but for consistency with analyses that use Pythia for the event generation the value of PARP(71) 


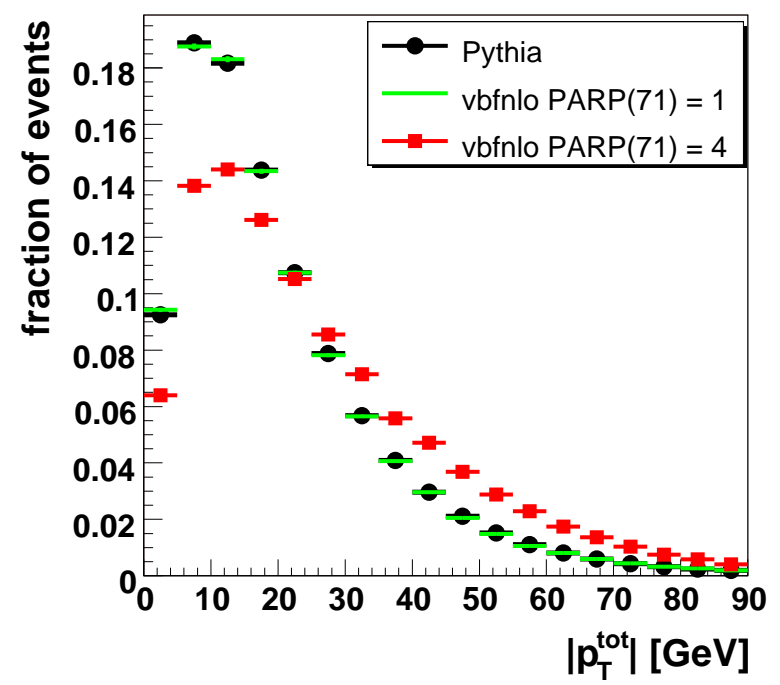

Figure 6: Distribution of $\left|p_{T}^{t o t}\right|$ in the channel $H \rightarrow W^{+} W^{-} \rightarrow l l \nu \nu$ after detector simulation and before cuts from events generated with Pythia and from events generated with vbfnlo for two different values of the parameter $\operatorname{PARP}(71)$ (see text).

is set to 1 in this study. Figures 7 and 8 show distributions of some cut variables for the channel $H \rightarrow W^{+} W^{-} \rightarrow l l \nu \nu$ from the final vbfnlo event sample with standard model couplings and from a control sample generated with Pythia. One can see that the remaining differences are small.

The event selection in the $H \rightarrow \tau^{+} \tau^{-}$channels is less sensitive to the value of PARP(71). With PARP $(71)=1$ the differences between vbfnlo and Pythia in these channels are small as in the case of $H \rightarrow W^{+} W^{-}$.

\section{Determination of the dominant coupling term}

After the discovery of a new scalar particle at the LHC a question of interest is by which of the 3 coupling terms presented in section 2 the coupling to weak bosons is described. By distinguishing between the $\mathrm{CP}$ even and the $\mathrm{CP}$ odd coupling terms one obtains information about the $\mathrm{CP}$ quantum number of the particle and by distinguishing between the standard model coupling term and the anomalous coupling terms one can test whether the coupling exists at leading order or is mediated by particle loops. In this section a hypothesis test is described in which the shape of the distribution of the azimuthal angles between the tagging jets $\Delta \phi_{j j}$ is used, as suggested in [2], to distinguish between the different couplings. The method is applied to the case of a standard model Higgs boson for which the prospects for the experimental exclusion of the anomalous couplings are investigated.

\subsection{Implementation of the hypothesis test}

A $\chi^{2}$ test is performed in the distribution of the variable $\Delta \phi_{j j}$ after the event selection has been applied. Figure 9 shows distributions of $\Delta \phi_{j j}$ for signal events with the three different couplings and for all three channels. One can see that the distributions are similar for the three different channels, the main difference being that large values of $\Delta \phi_{j j}$ are somewhat suppressed by the selection cuts in the $H \rightarrow \tau^{+} \tau^{-}$channels. The distributions for the 

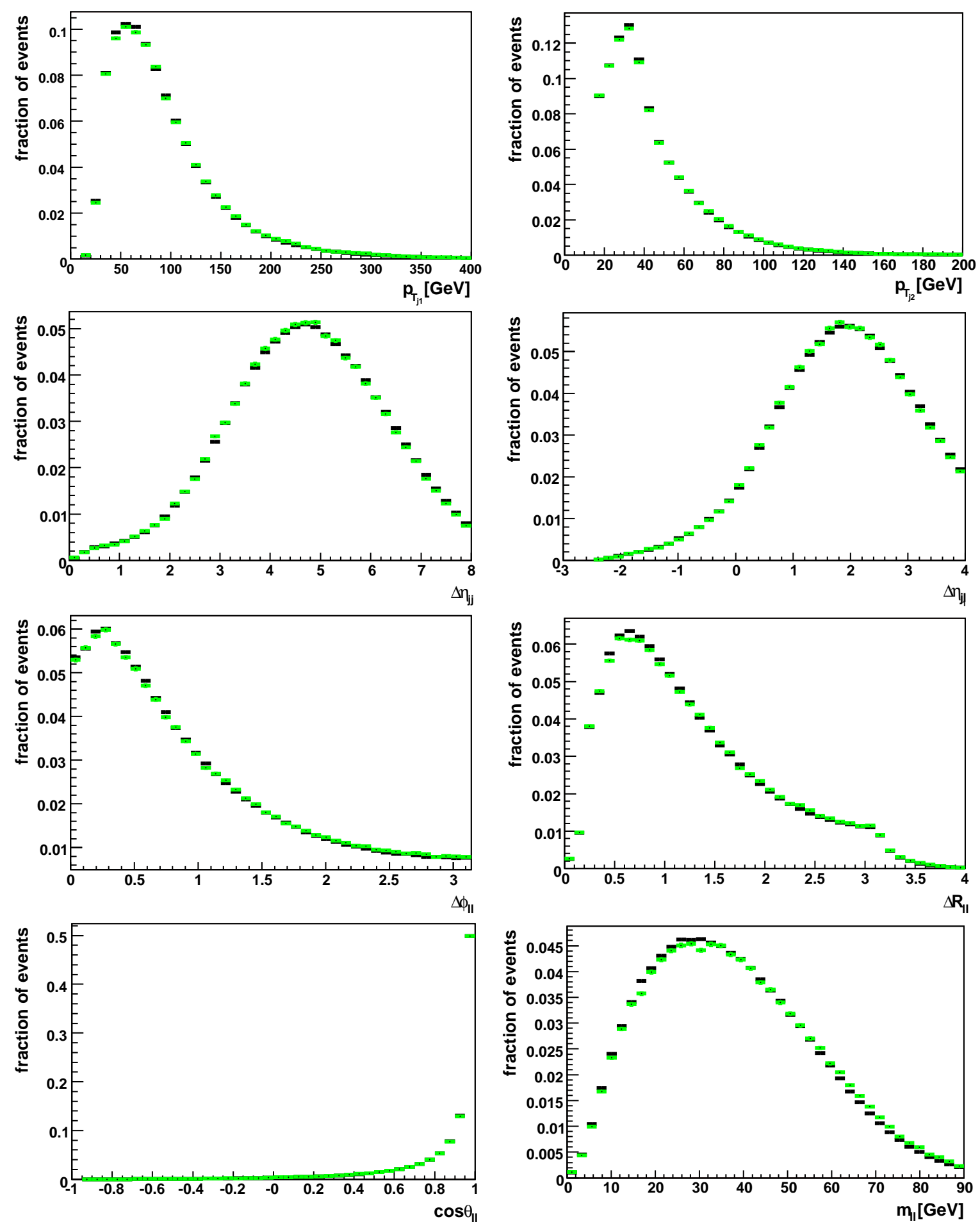

Figure 7: Distributions of some of the cut variables for the channel $H \rightarrow W^{+} W^{-} \rightarrow l l \nu \nu$ after the detector simulation and before cuts. The distributions from events generated with vbfnlo are drawn in black, the distributions from events generated with Pythia are drawn in green (light grey). 

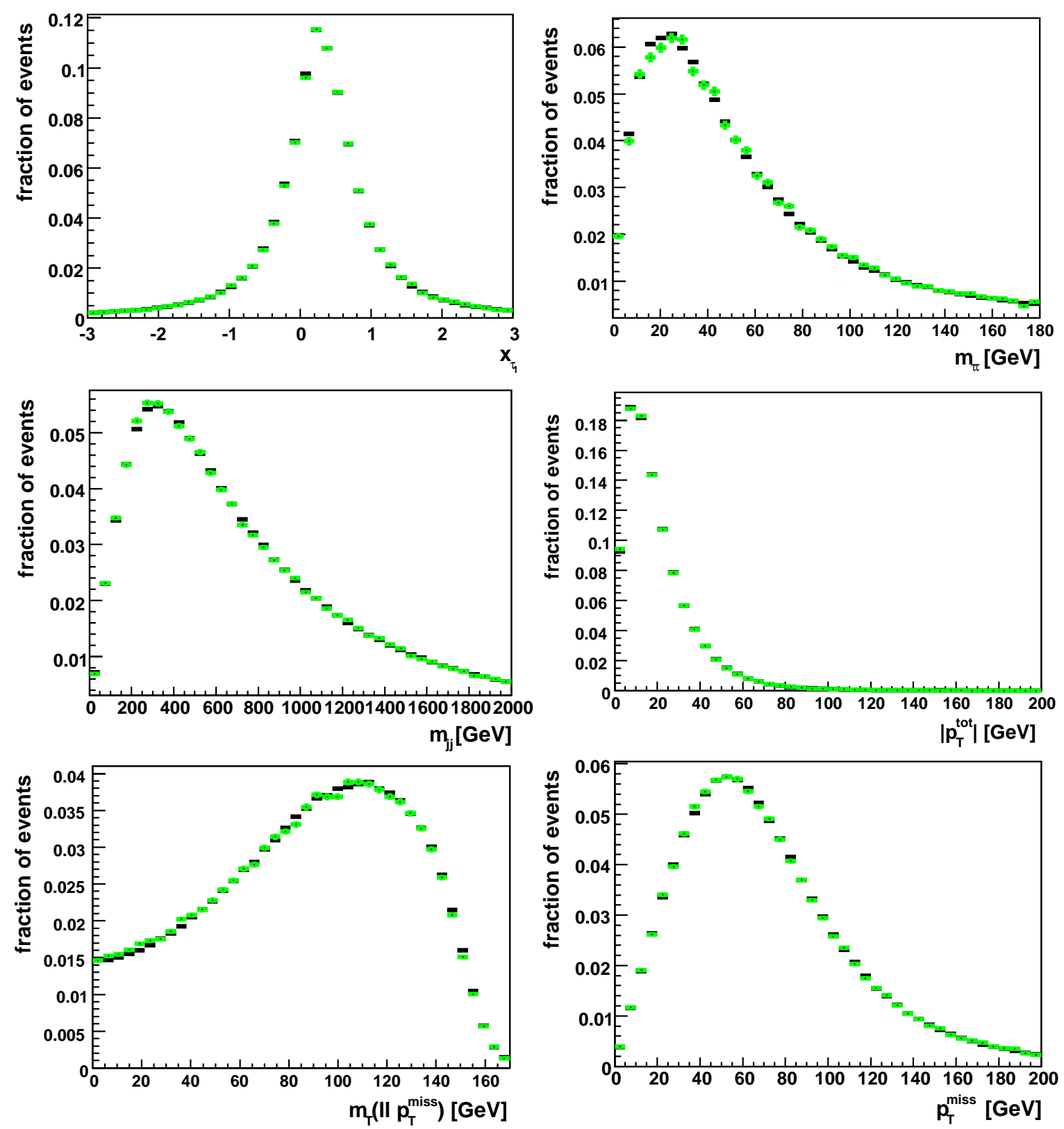

Figure 8: Distributions of some of the cut variables for the channel $H \rightarrow W^{+} W^{-} \rightarrow l l \nu \nu$ after the detector simulation and before cuts. The distributions from events generated with vbfnlo are drawn in black, the distributions from events generated with Pythia are drawn in green (light grey). 

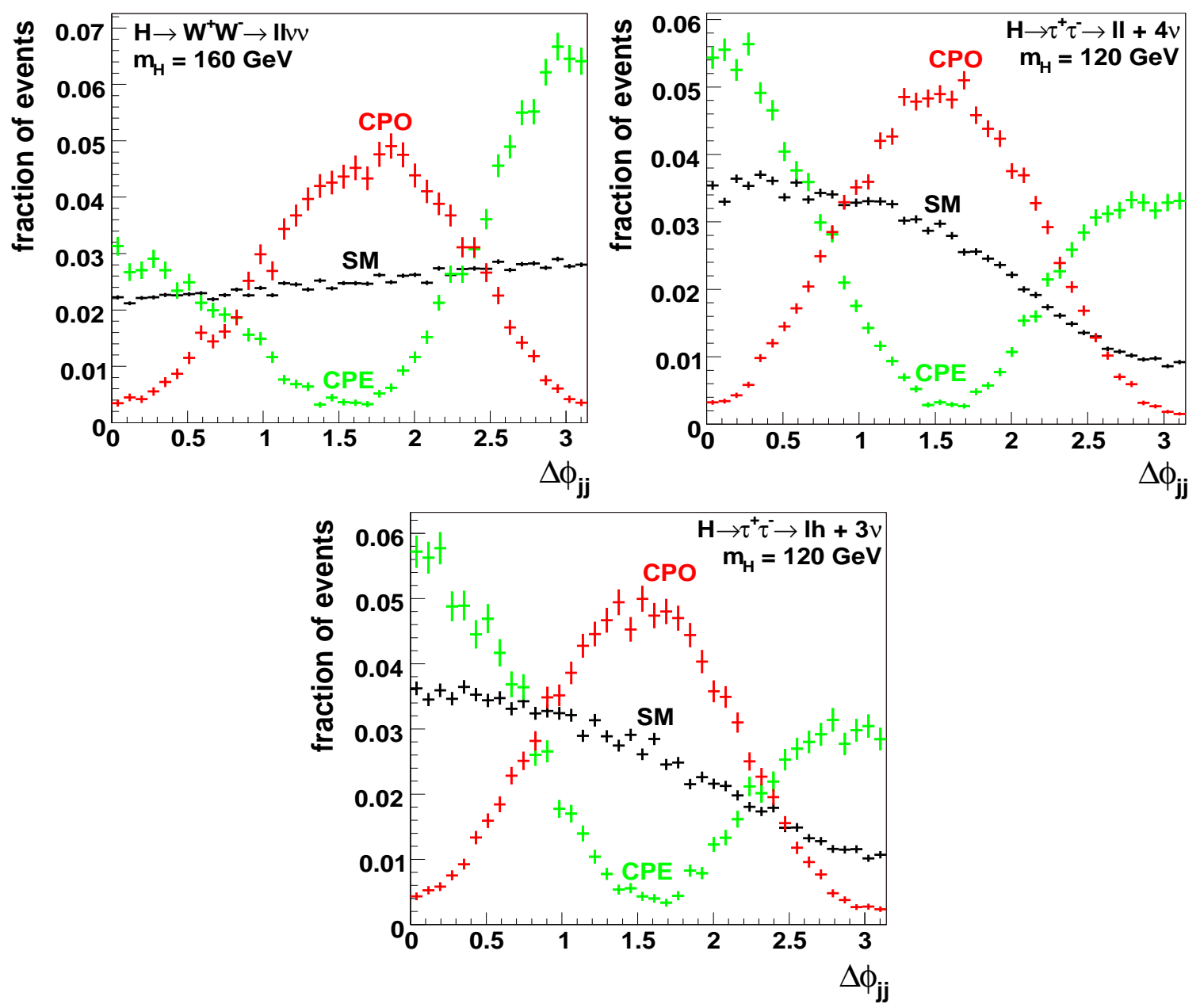

Figure 9: Distributions of the variable $\Delta \phi_{j j}$ with high statistics for signal events after all cuts have been applied. Distributions are shown for each of the three different couplings and each of the channels studied.

standard model case are fairly flat. The distributions for a CP odd anomalous coupling have a maximum at $\pi / 2$ and they are close to 0 for collinear $p_{T}$ of the tagging jets while the distributions for a $\mathrm{CP}$ even anomalous coupling show the opposite features.

From each signal and background event sample a reference distribution is calculated using the full statistics. The normalisation of the signal relative to the background in the standard model case follows from the standard model cross sections. For the case of anomalous couplings the normalisation is chosen such that the ratio of the number of signal events to the number of background events after cuts is the same as in the case of standard model couplings. With this choice of normalisation, the question that is investigated is whether for a scalar particle that has the expected experimental signature of a standard model Higgs boson the dominant coupling term can be determined. A prediction of the total cross section for the signal process for purely anomalous couplings is not necessary because only the shape of the signal distribution is used in the test. In an analysis with experimental data a different relative normalisation of signal and background may be preferred. This may be a value determined from experiment or from the predictions of a different model containing a scalar particle. 
background included:

\begin{tabular}{|cc|c|c|c|}
\hline \multicolumn{2}{|c|}{ hypothesis } & $H \rightarrow W^{+} W^{-} \rightarrow l l \nu \nu$ & $H \rightarrow \tau^{+} \tau^{-} \rightarrow l l+4 \nu$ & $H \rightarrow \tau^{+} \tau^{-}$combined \\
\hline $10 \mathrm{fb}^{-1}$ & $\mathrm{CPE}$ & $2.8 \cdot 10^{-4} \widehat{=} 3.6 \sigma$ & & \\
& $\mathrm{CPO}$ & $2.1 \cdot 10^{-10} \hat{=} 6.4 \sigma$ & & \\
$30 \mathrm{fb}^{-1}$ & $\mathrm{CPE}$ & $<10^{-15} \widehat{=}>7.7 \sigma$ & $6.2 \cdot 10^{-3} \widehat{=} 2.7 \sigma$ & $2.0 \cdot 10^{-4} \widehat{=} 3.7 \sigma$ \\
& $\mathrm{CPO}$ & $<10^{-15} \widehat{=}>7.7 \sigma$ & $6.6 \cdot 10^{-1} \widehat{=} 0.44 \sigma$ & $4.5 \cdot 10^{-1} \widehat{=} 0.76 \sigma$ \\
\hline
\end{tabular}

background not included, for comparison:

\begin{tabular}{|cc|c|c|c|}
\hline \multicolumn{2}{|c|}{ hypothesis } & $H \rightarrow W^{+} W^{-} \rightarrow l l \nu \nu$ & $H \rightarrow \tau^{+} \tau^{-} \rightarrow l l+4 \nu$ & $H \rightarrow \tau^{+} \tau^{-}$combined \\
\hline $10 \mathrm{fb}^{-1}$ & $\mathrm{CPE}$ & $3.3 \cdot 10^{-14} \hat{=} 7.6 \sigma$ & & \\
& $\mathrm{CPO}$ & $<10^{-15} \widehat{=}>7.7 \sigma$ & & \\
$30 \mathrm{fb}^{-1}$ & $\mathrm{CPE}$ & $<10^{-15} \hat{=}>7.7 \sigma$ & $2.2 \cdot 10^{-8} \widehat{=} 5.6 \sigma$ & $4.1 \cdot 10^{-9} \hat{=} 5.9 \sigma$ \\
& $\mathrm{CPO}$ & $<10^{-15} \hat{=}>7.7 \sigma$ & $7.2 \cdot 10^{-1} \hat{=} 0.36 \sigma$ & $3.5 \cdot 10^{-1} \hat{=} 0.93 \sigma$ \\
\hline
\end{tabular}

Table 9: Results of the $\chi^{2}$ test for one standard model pseudo-data sample per channel and integrated luminosity studied for the hypotheses of purely $\mathrm{CP}$ even and purely $\mathrm{CP}$ odd anomalous couplings (CPO). The upper table shows the results for samples in which background contributions are included. The lower table gives the values for the same signal events without background contributions for comparison. For the $H \rightarrow \tau^{+} \tau^{-}$case results are given for the channel $H \rightarrow \tau^{+} \tau^{-} \rightarrow l l+4 \nu$ and for both $H \rightarrow \tau^{+} \tau^{-}$channels combined.

The hypothesis test is performed on Monte Carlo pseudo-data samples containing signal events with standard model couplings and standard model background events corresponding to the expected amount of data after the first few years of data taking at the LHC at low luminosity. For the channel $H \rightarrow W^{+} W^{-} \rightarrow l l \nu \nu$ pseudo-data samples containing both standard model Higgs boson and background contributions corresponding to integrated luminosities of $10 \mathrm{fb}^{-1}$ and $30 \mathrm{fb}^{-1}$ are formed. For the $H \rightarrow \tau^{+} \tau^{-}$channels pseudo-data samples are formed only for $30 \mathrm{fb}^{-1}$. The pseudo-data samples are built from subsamples of the complete Monte Carlo samples from which the reference distributions are also calculated. Since the complete samples contain at least by a factor of 30 more events than the pseudodata samples the pseudo-data samples are assumed to be sufficiently statistically independent from the complete samples. Furthermore, the number of events in the complete samples is assumed to be sufficiently large with respect to the number of events in the pseudo-data samples for the statistical errors on the reference distributions to be negligible.

The $\chi^{2}$ is calculated for the number of bin entries $n_{i}$ of a histogram of $\Delta \phi_{j j}$ for a pseudodata sample and the number of bin entries of a reference histogram $\nu_{i}$ as

$$
\chi^{2}=\sum_{i} \frac{\left(n_{i}-\frac{\nu_{i} n}{\nu}\right)^{2}}{\frac{\nu_{i} n}{\nu}}
$$

with $n=\sum n_{i}$ and $\nu=\sum \nu_{i}$.

\subsection{Application to a few individual pseudo-data samples}

Some features of the test can be illustrated with exemplary results for individual pseudo-data samples. Figure 10 shows the distributions of $\Delta \phi_{j j}$ for one pseudo-data sample per channel together with the reference distributions for anomalous couplings which are normalised to the number of events in the pseudo-data distribution. One can see that the number of events in the $H \rightarrow \tau^{+} \tau^{-}$channels is quite small even for $30 \mathrm{fb}^{-1}$. Hence, the test is performed in 


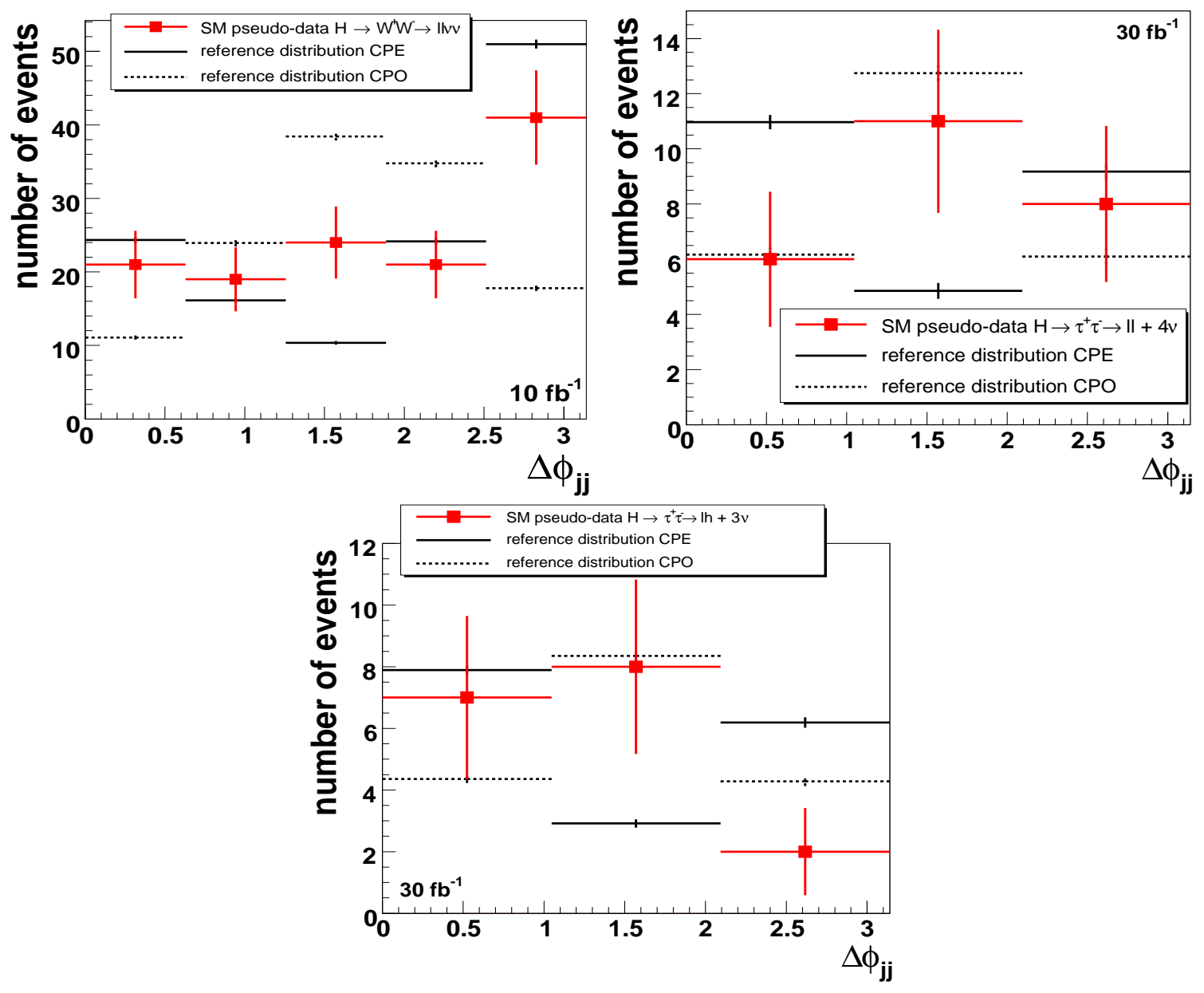

Figure 10: Distributions of $\Delta \phi_{j j}$ for one exemplary pseudo-data sample per channel. The reference distributions for the case of anomalous couplings shown are normalised to the number of entries of the pseudo-data histograms. Both pseudo-data and reference distributions include contributions from background processes.

3 bins. The results from the channels $H \rightarrow \tau^{+} \tau^{-} \rightarrow l l+4 \nu$ and $H \rightarrow \tau^{+} \tau^{-} \rightarrow l h+3 \nu$ are combined by adding the $\chi^{2}$ values for the histograms of both channels. In the channel $H \rightarrow W^{+} W^{-} \rightarrow l l \nu \nu$ the test is performed in 5 bins for $10 \mathrm{fb}^{-1}$ and in 10 bins for $30 \mathrm{fb}^{-1}$.

The results for the $\chi^{2}$ probability are shown in table 9 . For each $\chi^{2}$ probability the corresponding deviation from the mean of a Gaussian is calculated with a $\chi^{2}$ probability of $5.73 \cdot 10^{-7}$ corresponding to a deviation of $5 \sigma$. Results are given for the samples containing signal and background events and for comparison for the same signal events without background contributions. One can see that the $\chi^{2}$ probabilities differ by several orders of magnitude and that the corresponding deviation from the mean of a Gaussian changes by a factor of up to 2 when background events are neglected. For the tested samples a clear exclusion of the anomalous couplings is possible in the channel $H \rightarrow W^{+} W^{-} \rightarrow l l \nu \nu$ at $30 \mathrm{fb}^{-1}$ with good evidence already at $10 \mathrm{fb}^{-1}$. For the tested samples in the $H \rightarrow \tau^{+} \tau^{-}$ channels only the hypothesis of a $\mathrm{CP}$ even anomalous coupling can be excluded at a high confidence level. The results for $\mathrm{CP}$ even and $\mathrm{CP}$ odd anomalous couplings appear to be correlated. If the limit on one of the couplings is strong the pseudo-data distribution will 
typically look similar to the reference distribution of the other anomalous coupling. This can be seen for the examples in figure 10 in which the pseudo-data distributions for the channel $H \rightarrow W^{+} W^{-} \rightarrow l l \nu \nu$ look more similar to the CP even anomalous reference distribution while the pseudo-data distributions for the $H \rightarrow \tau^{+} \tau^{-}$channels both look more similar to the $\mathrm{CP}$ odd anomalous reference distribution.

\subsection{Application to many pseudo-data samples}

The $\chi^{2}$ test is repeated for many pseudo-data samples to obtain average values and distributions of the $\chi^{2}$ probabilities. As a first step disjoint subsamples are taken from the complete Monte Carlo samples. For an integrated luminosity of $10 \mathrm{fb}^{-1}$ the size of the Monte Carlo samples permits forming 149 subsamples in this way and for an integrated luminosity of $30 \mathrm{fb}^{-1} 49$ subsamples can be formed in the channel $H \rightarrow W^{+} W^{-} \rightarrow l l \nu \nu$ and 44 subsamples can be formed in the $H \rightarrow \tau^{+} \tau^{-}$channels. To increase the number of samples further subsamples are formed by resampling [22]. This method consists in taking events randomly from the complete Monte Carlo samples until the desired number is reached. An event that has been chosen is not marked and can be chosen again, even for the same pseudo-data sample. 10000 pseudo-data samples per channel are formed in this way. Effects of correlations between the samples due to events appearing in multiple samples and sometimes multiple times in the same sample are not investigated in this study.

The method is verified by calculating distributions of the $\chi^{2}$ probabilities with the reference distributions for standard model couplings. Since the hypothesis tested in this case is true the distributions of the $\chi^{2}$ probabilities must be flat within statistical fluctuations. The distributions are shown in figure 11 and one can see that they agree with the expectation.

Distributions of the $\chi^{2}$ probabilities for the hypotheses of CP even and CP odd anomalous couplings are shown in figures 12 and 13. From the distributions the probability of a pseudo-data sample to have a $\chi^{2}$ probability below a given threshold can be calculated. This can be interpreted as an estimate of the probability to obtain a $\chi^{2}$ probability at the threshold value or below from a standard model event sample of the given integrated luminosity at the ATLAS experiment. These probabilities are given in table 10 for the pseudo-data samples from resampling and for threshold values of the $\chi^{2}$ probability of $5 \%$ and a value corresponding to $5 \sigma$. In addition the median $\chi^{2}$ probability and the corresponding deviation from the mean of a Gaussian distribution in standard deviations are given for the channels in which more than half of the pseudo-data samples have a $\chi^{2}$ probability above $10^{-15}$ which is the limit of the numerical precision of the calculation.

\subsubsection{Results of the hypothesis test}

According to the results in table 10 it appears to be possible to exclude purely anomalous $\mathrm{CP}$ even and purely anomalous $\mathrm{CP}$ odd couplings for a standard model Higgs boson at $m_{H}=160 \mathrm{GeV}$ in the channel $H \rightarrow W^{+} W^{-} \rightarrow l l \nu \nu$ already from $10 \mathrm{fb}^{-1}$ of low luminosity data at a confidence level corresponding to approximately $5 \sigma$. For a standard model Higgs boson at $m_{H}=120 \mathrm{GeV}$ in the $H \rightarrow \tau^{+} \tau^{-}$channels the expected limits for the exclusion are weaker. However, on average an exclusion with a confidence level corresponding to $2 \sigma$ or more is expected for an integrated luminosity of $30 \mathrm{fb}^{-1}$.

\subsection{Effects of uncertainties on the number of background events}

The total number of background events in a data sample from the ATLAS detector is estimated in [1] to be measurable with an uncertainty of $\pm 10 \%$. The effect on the $\chi^{2}$ test of 

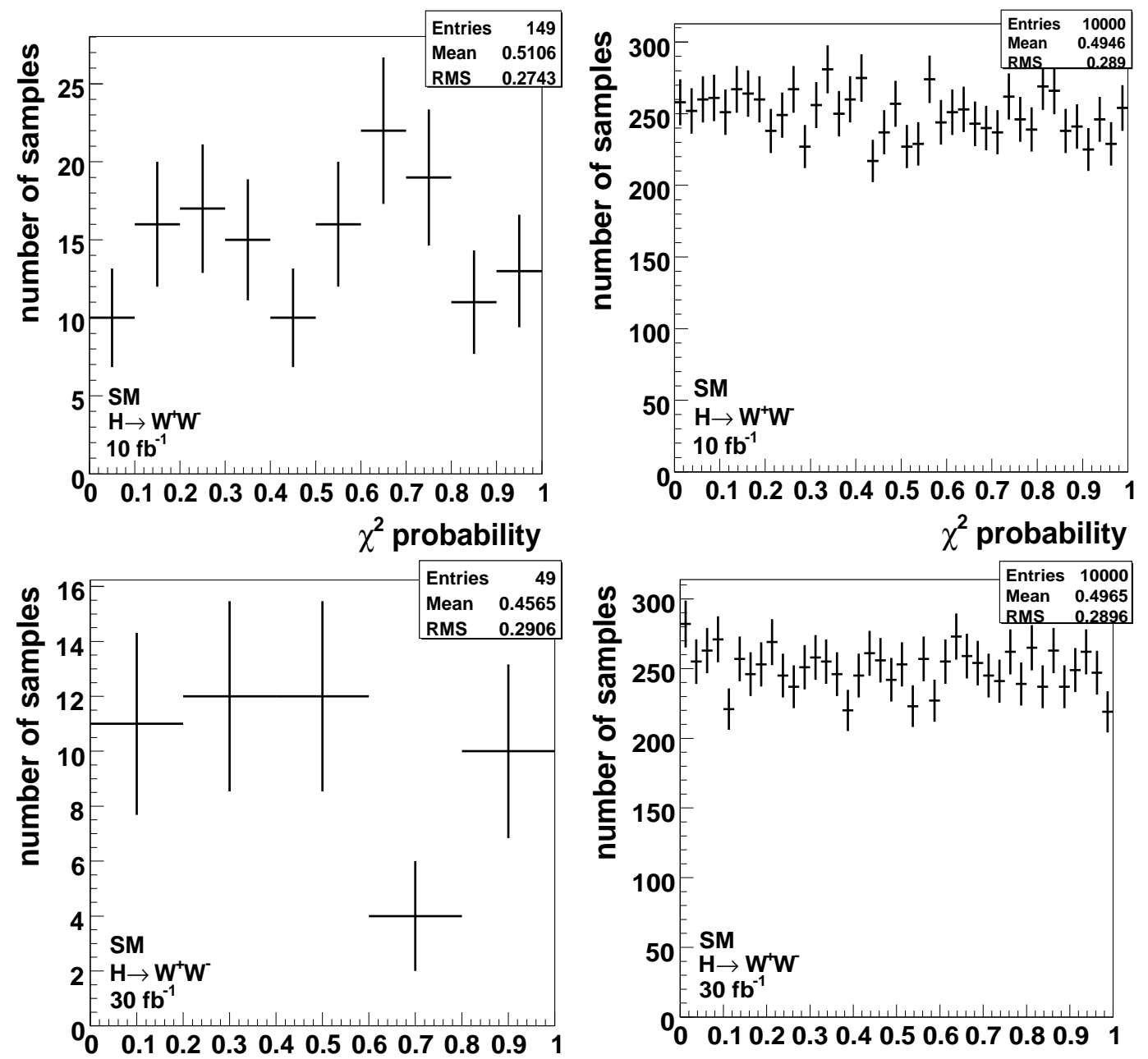

$\chi^{2}$ probability
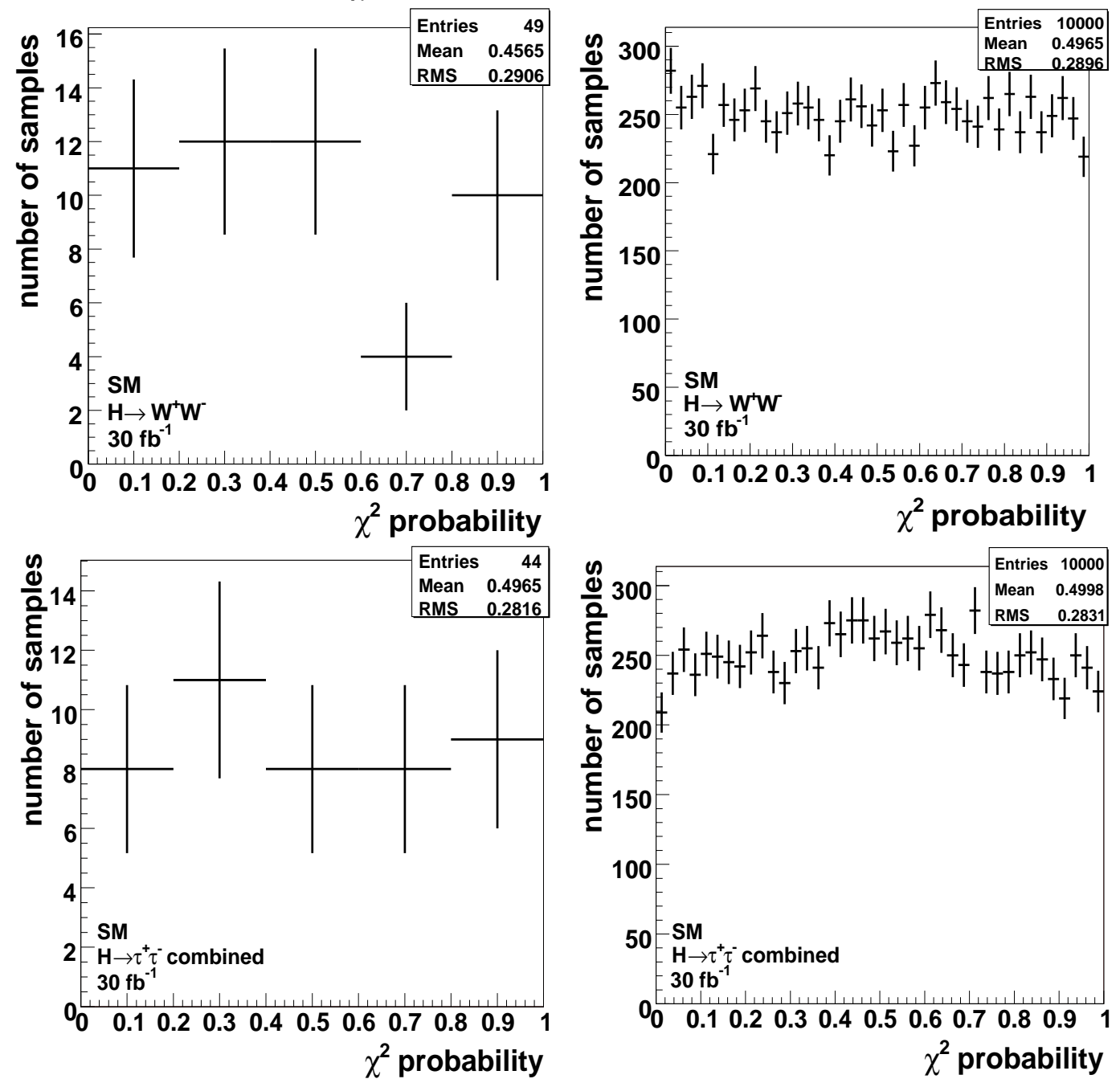

Figure 11: Control distributions of the $\chi^{2}$ probabilities for the standard model hypothesis. Distributions for disjoint subsamples are shown on the left and distributions for pseudo-data samples from resampling are shown on the right. 

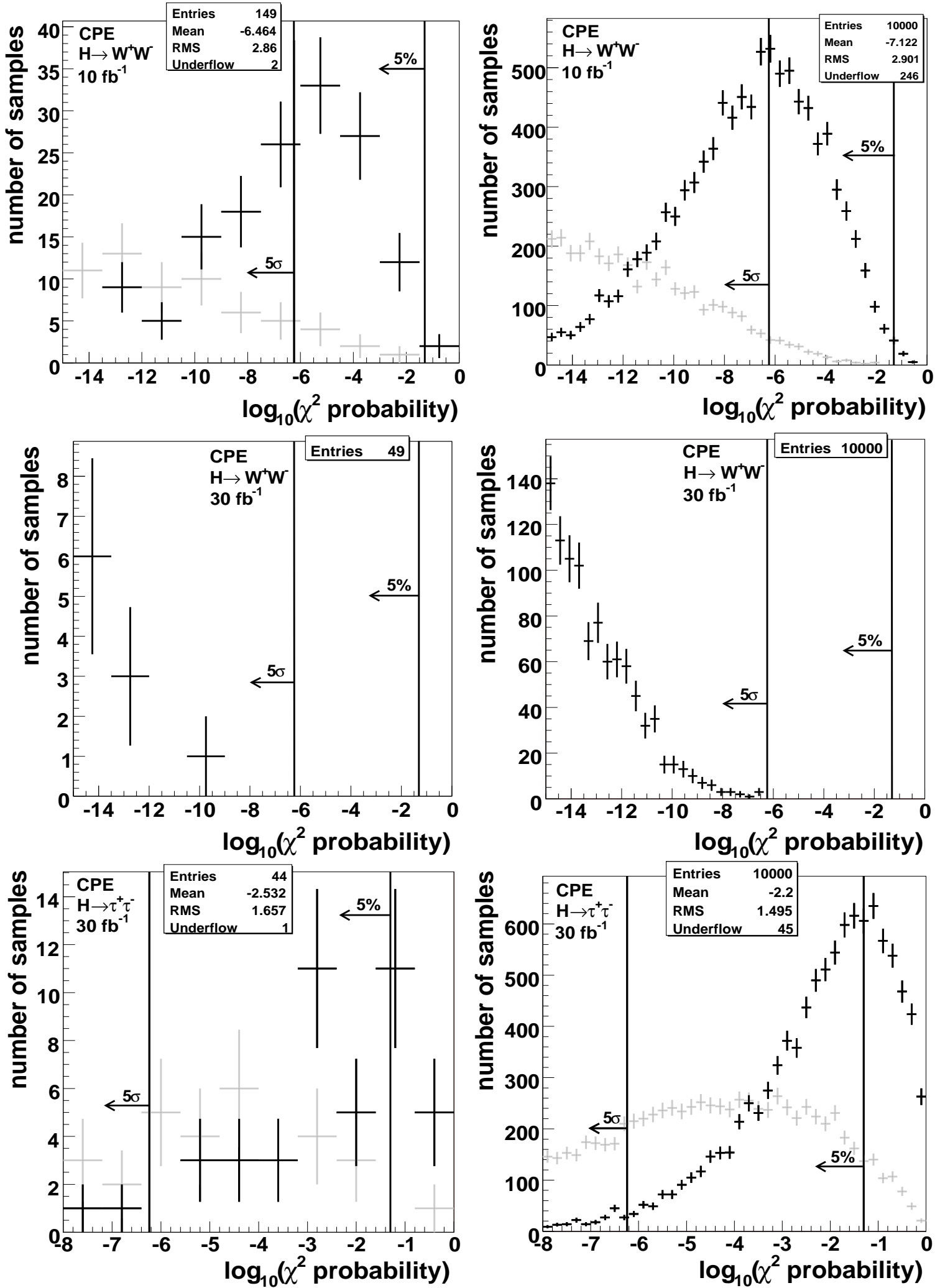

Figure 12: Distributions of $\chi^{2}$ probabilities for the hypothesis of a purely anomalous CP even coupling. Distributions for disjoint subsamples are shown on the left and distributions for pseudo-data samples from resampling are shown on the right. For comparison distributions in which background contributions have been neglected are shown in grey. The distributions are only shown down to a value of $10^{-15}$ which is the limit of the numerical precision of the calculation. 

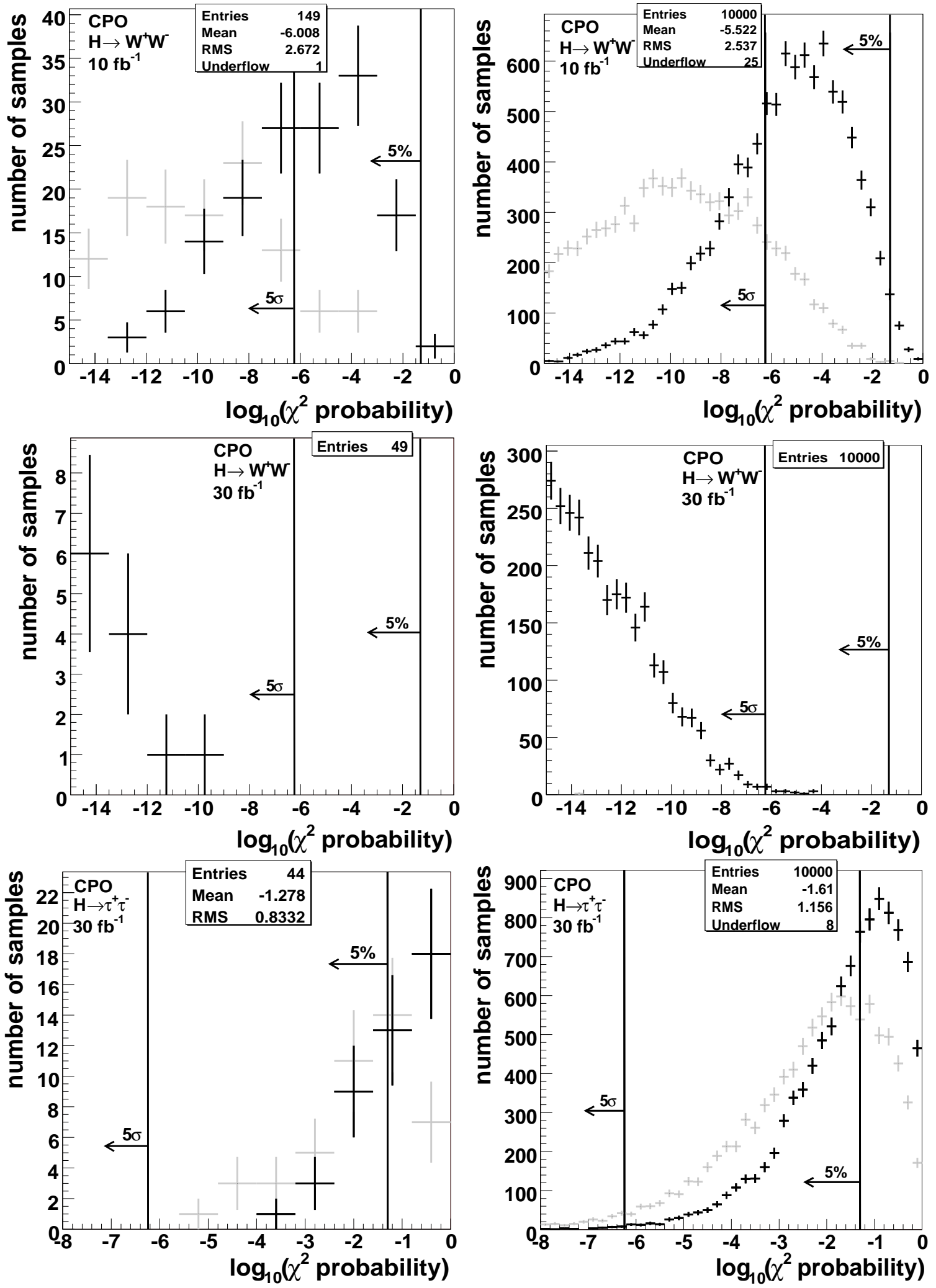

Figure 13: Distributions of $\chi^{2}$ probabilities for the hypothesis of a purely anomalous CP odd coupling. Distributions for disjoint subsamples are shown on the left and distributions for pseudo-data samples from resampling are shown on the right. For comparison distributions in which background contributions have been neglected are shown in grey. The distributions are only shown down to a value of $10^{-15}$ which is the limit of the numerical precision of the calculation. 


\begin{tabular}{|c|c|c|c|c|}
\hline \multirow{2}{*}{$\begin{array}{l}\text { integrated luminosity, } \\
\text { hypothesis tested }\end{array}$} & \multicolumn{2}{|c|}{ probability for } & \multicolumn{2}{|c|}{ median } \\
\hline & $>5 \sigma$ & $<5 \%$ & $\chi^{2}$-prob. & ev. in $\sigma$ \\
\hline \multicolumn{5}{|l|}{$H \rightarrow W^{+} W^{-} \rightarrow l l \nu \nu$} \\
\hline $10 \mathrm{fb}^{-1}$ & $59 \%$ & $100 \%$ & $1.3 \cdot 10^{-7}$ & $5.3 \sigma$ \\
\hline $\mathrm{CPO}$ & $35 \%$ & $98 \%$ & $6.0 \cdot 10^{-6}$ & $4.5 \sigma$ \\
\hline \multirow[t]{2}{*}{$30 \mathrm{fb}^{-1}$} & $100 \%$ & $100 \%$ & - & - \\
\hline & $100 \%$ & $100 \%$ & - & - \\
\hline \multicolumn{5}{|l|}{$H \rightarrow \tau^{+} \tau^{-}$combined } \\
\hline $30 \mathrm{fb}^{-1}$ & $2 \%$ & $68 \%$ & $1.2 \cdot 10^{-2}$ & $2.5 \sigma$ \\
\hline $\mathrm{CPO}$ & $0 \%$ & $52 \%$ & $4.3 \cdot 10^{-2}$ & $2.0 \sigma$ \\
\hline
\end{tabular}

Table 10: The first two columns show the probabilities for a standard model pseudo-data sample in tests of the hypotheses of purely CP even (CPE) and CP odd (CPO) anomalous couplings to have a $\chi^{2}$ probability below $5 \%$ or $5.7 \cdot 10^{-7}=5 \sigma$. The third and fourth columns show the median $\chi^{2}$ probabilities and the corresponding deviations from the mean of a Gaussian distribution in standard deviations.

an uncertainty of the background normalisation in the data of this size is studied by repeating the $\chi^{2}$ test on pseudo-data samples from resampling with modified average numbers of background events. In a first calculation, the average numbers of events for all background processes in the pseudo-data samples are varied by $+10 \%$. In a second calculation they are varied by $-10 \%$. The reference distributions are left unchanged.

One can see from the results given in table 11 that the median $\chi^{2}$ probability for the channel $H \rightarrow W^{+} W^{-} \rightarrow l l \nu \nu$ is smaller when the average number of background events in the pseudo-data samples is larger than the number assumed for calculating the reference distributions. This is explained by the fact that the signal and background distributions have similar shapes for standard model couplings and a standard model pseudo-data distribution with a larger number of signal or background events can be distinguished from a distribution for anomalous couplings with a higher confidence level. This does not represent a real improvement of the sensitivity of the measurement but a measurement error induced by the error on the background normalisation. If the background in the data is underestimated, the sensitivity for the exclusion of anomalous couplings is slightly overestimated. For the case of less background events the median $\chi^{2}$ probability increases accordingly. In the $H \rightarrow \tau^{+} \tau^{-}$ channels this effect is present but very small.

One can see that the median $\chi^{2}$ probability changes by a factor of less than 1.8 with respect to the case of a correct background prediction. The change in the corresponding number of standard deviations is 0.1 or less in all cases. Thus, the uncertainty on the measured number of background events in the data has only a small effect on the results of the $\chi^{2}$ test.

\section{Sensitivity to a small CP even anomalous coupling contribution}

Once the dominant term of the coupling of a new scalar particle to weak bosons has been determined a question will be whether a small contribution of another coupling term exists. In this section a likelihood fit is used to estimate the sensitivity of the ATLAS experiment to small $\mathrm{CP}$ even anomalous couplings that may be present in addition to the standard model couplings. The likelihood fit is not expected to be sensitive to a contribution by CP 
Results for $+10 \%$ background events:

\begin{tabular}{|cc|c|c|c|c|}
\hline \multirow{2}{*}{$\begin{array}{c}\text { integrated luminosity, } \\
\text { hypothesis tested }\end{array}$} & \multicolumn{2}{|c|}{ probability for } & \multicolumn{2}{|c|}{ median } \\
\hline$H \rightarrow W^{+} W^{-} \rightarrow l l \nu \nu$ & & $<5 \%$ & $\chi^{2}$-prob. & dev. in $\sigma$ \\
\hline$H \mathrm{fb}^{-1}$ & CPE & $61 \%$ & $100 \%$ & $7.6 \cdot 10^{-8}$ & $5.4 \sigma$ \\
& CPO & $37 \%$ & $98 \%$ & $4.4 \cdot 10^{-6}$ & $4.6 \sigma$ \\
$30 \mathrm{fb}^{-1}$ & CPE & $100 \%$ & $100 \%$ & - & - \\
& CPO & $100 \%$ & $100 \%$ & - & - \\
\hline$H \rightarrow \tau^{+} \tau^{-}$combined & & & & \\
$30 \mathrm{fb}^{-1}$ & CPE & $3 \%$ & $68 \%$ & $1.2 \cdot 10^{-2}$ & $2.5 \sigma$ \\
& CPO & $0 \%$ & $52 \%$ & $4.3 \cdot 10^{-2}$ & $2.0 \sigma$ \\
\hline
\end{tabular}

Results for $-10 \%$ background events:

\begin{tabular}{|c|c|c|c|c|}
\hline $\begin{array}{l}\text { integrated luminosity, } \\
\text { hypothesis tested }\end{array}$ & \multicolumn{2}{|c|}{ probability for } & $\begin{array}{r}\mathrm{m \epsilon} \\
\chi^{2} \text {-prob. }\end{array}$ & ev. in $\sigma$ \\
\hline$H \rightarrow W^{+} W^{-} \rightarrow l l \nu \nu$ & & & & \\
\hline $10 \mathrm{fb}^{-1}$ & $57 \%$ & $100 \%$ & $1.7 \cdot 10^{-7}$ & $5.2 \sigma$ \\
\hline $\mathrm{CPO}$ & $33 \%$ & $98 \%$ & $9.1 \cdot 10^{-6}$ & $4.4 \sigma$ \\
\hline $30 \mathrm{fb}^{-1}$ & $100 \%$ & $100 \%$ & - & - \\
\hline $\mathrm{CPO}$ & $100 \%$ & $100 \%$ & - & - \\
\hline$H \rightarrow \tau^{+} \tau^{-}$combined & 2 & 680 & 13 & 2 \\
\hline $\mathrm{CPO}$ & $0 \%$ & $52 \%$ & $4.3 \cdot 10^{-2}$ & $2.0 \sigma$ \\
\hline
\end{tabular}

Table 11: Results of the $\chi^{2}$ test applied to standard model pseudo-data samples from resampling for tests of the hypotheses of purely $\mathrm{CP}$ even $(\mathrm{CPE})$ and purely $\mathrm{CP}$ odd (CPO) anomalous couplings, with the average numbers of events for all background processes varied by $\pm 10 \%$ with respect to the expected numbers. Probabilities for a pseudo-data sample to have a $\chi^{2}$ probability below $5 \%$ or $5.7 \cdot 10^{-7} \widehat{=} 5 \sigma$ as well as median $\chi^{2}$ probabilities and the corresponding deviations from the mean of a Gaussian distribution in standard deviations are given.

odd anomalous couplings in addition to the standard model couplings and hence is not used to determine the sensitivity to a contribution by CP odd couplings. The reason for this expectation will be discussed later in this section.

\subsection{Implementation of the likelihood fit}

The azimuthal angle between the tagging jets $\Delta \phi_{j j}$ is used as the sensitive quantity in the likelihood fit. In the presence of small $\mathrm{CP}$ even anomalous couplings in addition to the standard model couplings the distribution of $\Delta \phi_{j j}$ is modified with respect to the standard model case by the interference term in the squared matrix element for Higgs boson production in WBF. Distributions of $\Delta \phi_{j j}$ for signal events with three different values of $g_{5 e}^{H Z Z}$ are shown in figure 14. As before, the ratio of anomalous $H W W$ couplings to anomalous $H Z Z$ couplings is kept fixed at $g_{5 e}^{H Z Z}=g_{5 e}^{H W W} \cos ^{2} \theta_{W}$. The values of the anomalous coupling constant chosen in figure 14 are close to the experimental limits given in table 3 for the $H \rightarrow \tau^{+} \tau^{-}$channels and they are well within the limits for the channel $H \rightarrow W^{+} W^{-} \rightarrow l l \nu \nu$. 


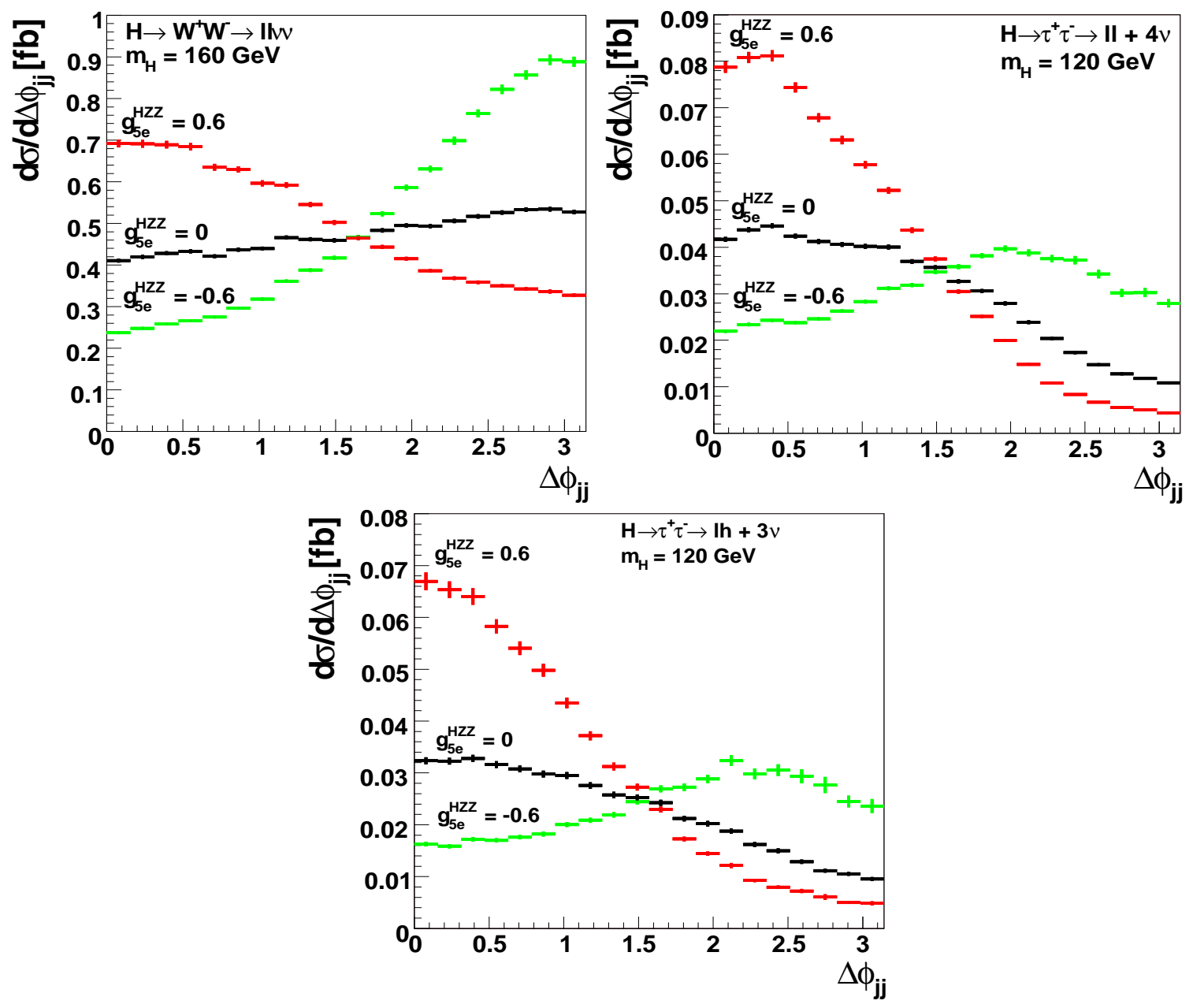

Figure 14: Distributions of $\Delta \phi_{j j}$ for signal events after all cuts have been applied. For each channel distributions are shown for three different values of the CP even anomalous coupling constant. The distributions were calculated by reweighting of events as described in section 6.2 .

Furthermore, the values are chosen such that the interference term gives a larger contribution than the squared anomalous term. One can see from figure 14 that the interference term is equal to 0 and changes its sign at about $\pi / 2$. The maximum of the distribution is located at large or small values of $\Delta \phi_{j j}$ depending on the sign of the anomalous couplings. While the interference term significantly influences the distribution of $\Delta \phi_{j j}$ it has only a small effect on the total cross section due to the asymmetry in the distribution. Thus, it is expected that the sensitivity to a small contribution by $\mathrm{CP}$ even anomalous couplings from an analysis of the shape of the distribution of $\Delta \phi_{j j}$ will be higher than from a measurement of the total cross section.

As pointed out in [2] interference effects between the CP odd anomalous couplings and standard model couplings do not show in the shape of the distribution of $\Delta \phi_{j j}$ used in this analysis. Hence, a likelihood fit in the shape of the distribution will probably not be sensitive to small CP odd anomalous couplings. A contribution of the CP odd anomalous couplings may be determined from a measurement of the total cross section. If the contribution is large enough the effect of the squared anomalous matrix element may be observable in the 
distribution of $\Delta \phi_{j j}$ with the shape as shown in section 5. Recently it has been suggested [24] to use a redefined $\Delta \phi_{j j}$ that can assume positive as well as negative values to study effects of small CP odd anomalous couplings. This is a possible topic for further research extending the analysis presented here.

The logarithm of the likelihood is calculated for the number of bin entries in a pseudo-data histogram $n_{i}$, the number of bin entries in a reference histogram $\nu_{i}$ and the number of bins $N$ as

$$
\ln L\left(g_{5 e}^{H Z Z}\right)=\sum_{i=1}^{N} n_{i} \ln \nu_{i}\left(g_{5 e}^{H Z Z}\right) .
$$

This form of the likelihood follows from the assumption of a multinomial distribution of the data. The total number of events is not a part of the prediction and the reference histogram is normalised to the data histogram before the likelihood is calculated.

If the total number of events depends on one or more of the arguments of the parameters to be estimated it can be advantageous to include the number of events in the prediction. In this case the distribution of the data is assumed to be given by a product of independent Poisson distributions in each bin. The logarithm of the resulting so-called extended likelihood is given by

$$
\ln L\left(g_{5 e}^{H Z Z}\right)=-\nu_{t o t}\left(g_{5 e}^{H Z Z}\right)+\sum_{i=1}^{N} n_{i} \ln \nu_{i}\left(g_{5 e}^{H Z Z}\right)
$$

with $\nu_{t o t}=\sum \nu_{i}$. For the calculation of the extended likelihood the reference histogram is normalised to the expected total number of events.

\subsection{Reweighting of events}

The calculation of the likelihood of equations (24) and (25) requires reference distributions of $\Delta \phi_{j j}$ for continuous values of the coupling constant $g_{5 e}^{H Z Z}$. Instead of generating large event samples for several values of $g_{5 e}^{H Z Z}$ the reference distributions are calculated by generating a large Monte Carlo event sample with standard model couplings and reweighting the events. Instead of filling histograms with a weight of 1 for each event the events are assigned a weight given by the ratio of the differential cross section at the phase space point of the event with standard model and anomalous couplings to the differential cross section with standard model couplings only. The ratio of the differential cross sections is given by the ratio of the squared matrix element with standard model and anomalous couplings to the squared matrix element with standard model couplings only. If the Monte Carlo event sample with standard model couplings contains enough events to sufficiently populate all regions of phase space that should be populated after the reweighting, all distributions are expected to have the same shape and normalisation as for events generated with anomalous couplings. The only difference expected is that the statistical errors will be different. The statistical error of the sum $W$ of weights $w_{i}$ in a bin is given by

$$
\sigma^{2}(W)=\sum_{i} w_{i}^{2}
$$

where the sum runs over all events in the bin [25]. If, as expected, all distributions have the correct shape and normalisation, the event selection can be applied as in the case of unweighted events.

The event weights are calculated for the parton momenta in the output files of the program vbfnlo. Subroutines of vbfnlo are used for the calculation of the matrix elements. The method of reweighting is tested by comparing the distributions from reweighted events to 

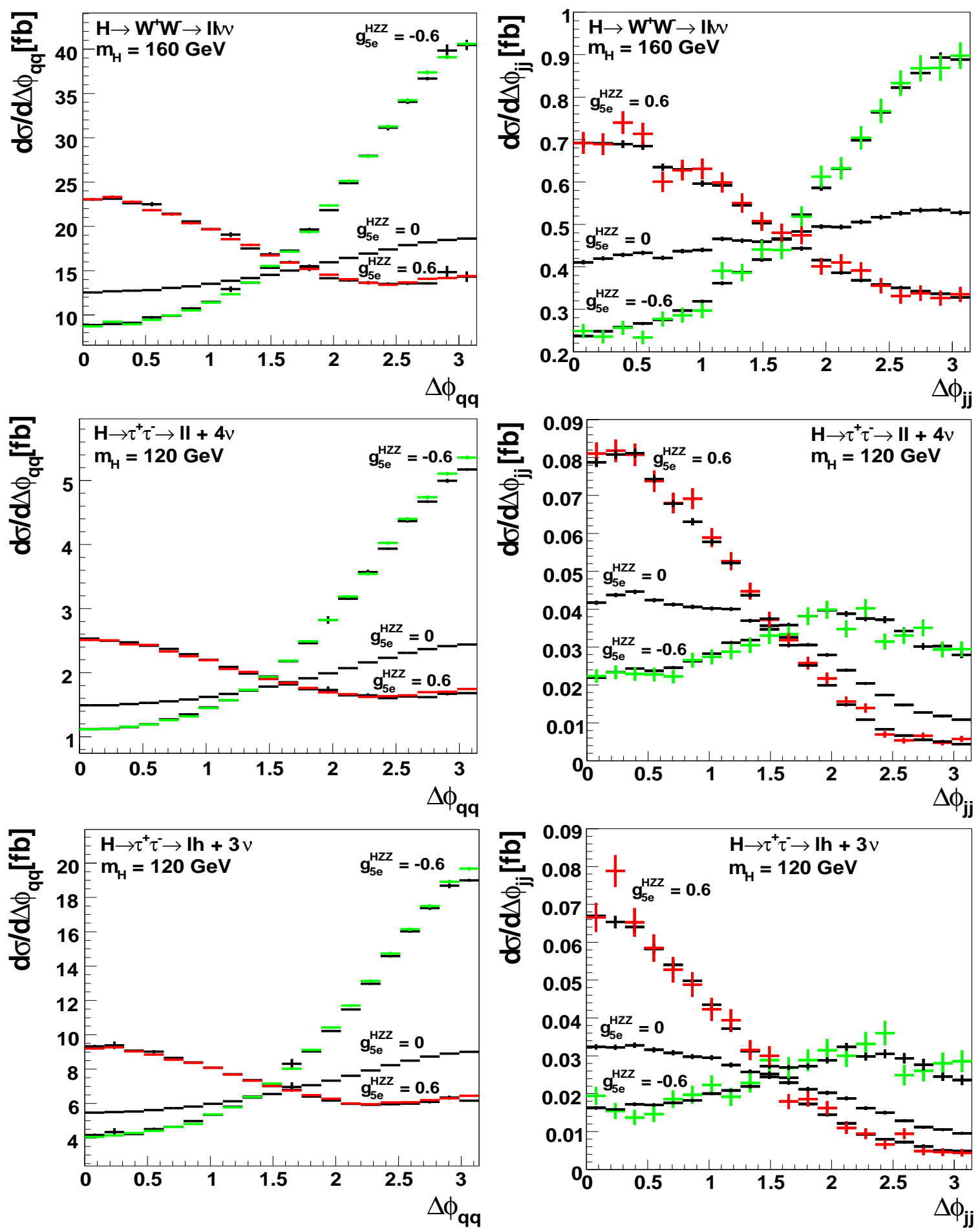

Figure 15: Distributions from signal events of the azimuthal angle between the scattered quarks at parton level on the left and of the azimuthal angle between the tagging jets after the event selection on the right for each of the three channels studied and three different values of the anomalous coupling constant $g_{5 e}^{H Z Z}$. Distributions calculated by reweighting are shown in black. For comparison distributions calculated from events that were generated with anomalous couplings are shown in colour (dark/light grey). The coloured (grey) distributions are normalised to the cross section for the respective combination of couplings. For the black distributions the number of events is normalised to the standard model cross section while the final normalisation of the histogram follows from the weights. 

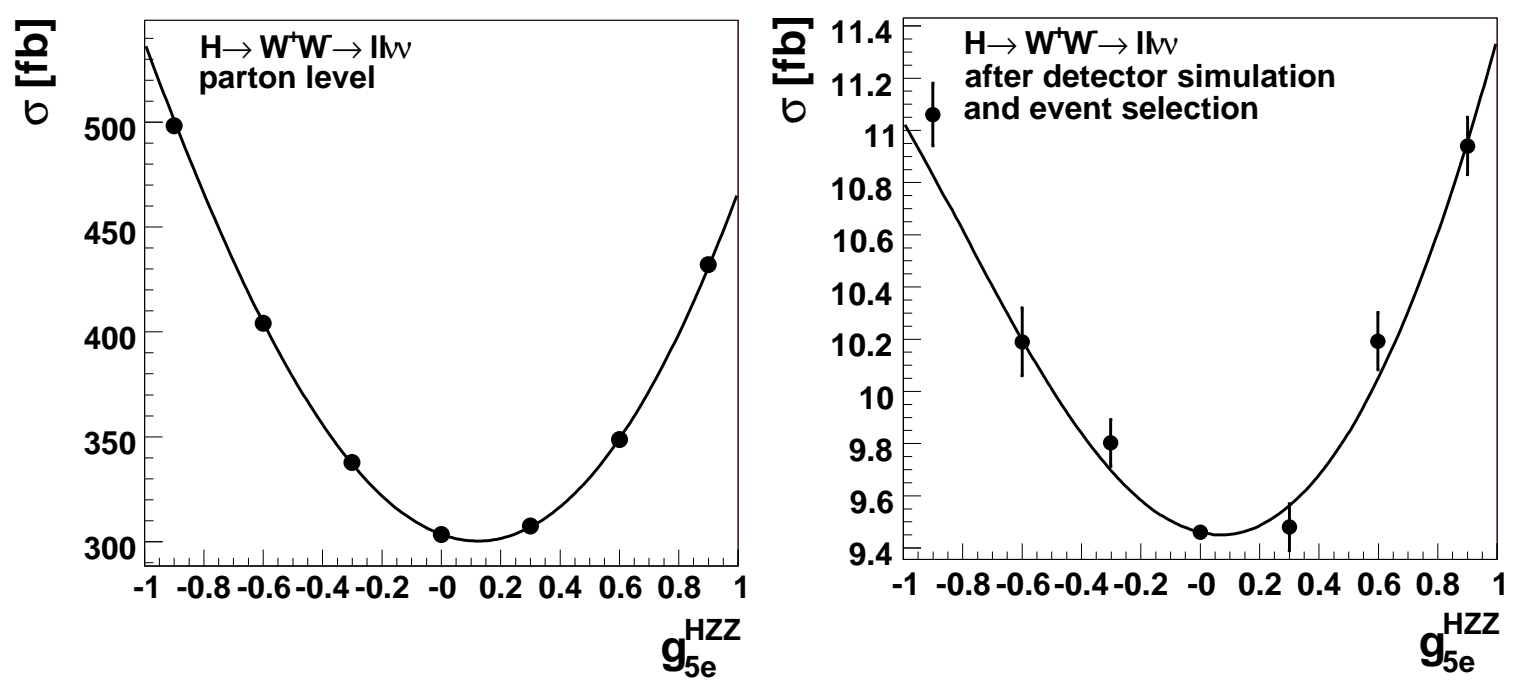

Figure 16: Total cross section of the signal process in the channel $H \rightarrow W^{+} W^{-} \rightarrow l l \nu \nu$ as a function of the anomalous coupling constant $g_{5 e}^{H Z Z}$. The curves were calculated by reweighting of events. The points were calculated from events generated at the respective values of $g_{5 e}^{H Z Z}$. The cross section at parton level is shown on the left and the cross section after the detector simulation and event selection is shown on the right. The errors on the points in the right figure are the binomial errors that arise from the reconstruction and selection efficiencies.

\begin{tabular}{|c|c|c|c|c|}
\hline & \multicolumn{2}{|c|}{$\sigma[\mathrm{fb}]$ at parton level } & \multicolumn{2}{c|}{$\sigma[\mathrm{fb}]$ after ATLFAST and cuts } \\
\hline$g_{5 e}^{\mathrm{HZZ}}$ & generated & reweighted & generated & reweighted \\
\hline-0.9 & $498.4 \pm 0.9$ & $499.1 \pm 2.3$ & $11.06 \pm 0.12$ & $10.82 \pm 0.05$ \\
-0.6 & $401.5 \pm 0.9$ & $403.0 \pm 1.0$ & $10.19 \pm 0.13$ & $10.19 \pm 0.03$ \\
-0.3 & $335.6 \pm 0.9$ & $336.1 \pm 0.3$ & $9.80 \pm 0.09$ & $9.69 \pm 0.02$ \\
0.0 & 303.3 & 303.3 & $9.46 \pm 0.02$ & $9.46 \pm 0.02$ \\
0.3 & $307.3 \pm 0.9$ & $307.3 \pm 0.2$ & $9.48 \pm 0.09$ & $9.57 \pm 0.02$ \\
0.6 & $348.6 \pm 0.9$ & $350.1 \pm 0.7$ & $10.19 \pm 0.11$ & $10.06 \pm 0.03$ \\
0.9 & $431.4 \pm 0.9$ & $432.5 \pm 1.5$ & $10.94 \pm 0.11$ & $10.97 \pm 0.04$ \\
\hline
\end{tabular}

Table 12: Values of the total cross section for the signal process in the channel $H \rightarrow$ $W^{+} W^{-} \rightarrow l l \nu \nu$ from reweighted events and for comparison from generated events. Cross section values at parton level are shown on the left and cross section values after the detector simulation and event selection are shown on the right. The statistical errors on the values from generated events at parton level were calculated from the errors given by the program vbfnlo. The statistical errors on the values from reweighting were calculated using the error on the sum of weights from equation 26. The statistical errors on the values from generated events after the event selection are the binomial errors given by the reconstruction and selection efficiencies. 
the distributions from events that were generated with anomalous couplings. Distributions at parton level and after the detector simulation and event selection for three different values of $g_{5 e}^{H Z Z}$ and for all three channels are shown in figure 15. One can see from the figure that the distributions from generated and from reweighted events agree with each other both in shape and normalisation. Small differences are observed in the $H \rightarrow \tau^{+} \tau^{-}$at parton level for large $\Delta \phi_{q q}$.

The correctness of the normalisation of the histograms from reweighted events is explicitly checked in the channel $H \rightarrow W^{+} W^{-} \rightarrow l l \nu \nu$ by comparing the prediction from reweighted events with the prediction from additional event samples that were generated for 6 values of $g_{5 e}^{H Z Z}$ between -0.9 and 0.9 . The calculated total cross section as a function of $g_{5 e}^{H Z Z}$ is shown in figure 16 both at parton level and after the detector simulation and event selection. The values of the cross section at the points of $g_{5 e}^{H Z Z}$ at which the additional event samples were generated are given in table 12 . One can see from figure 16 and table 12 that within statistical fluctuations the reweighting method leads to the same total cross section prediction as the direct Monte Carlo calculation with anomalous couplings, both at parton level and after the detector simulation and event selection. Furthermore, one can see that the dependence of the total cross section on the anomalous coupling constant $g_{5 e}^{H Z Z}$ is weaker after the detector simulation and event selection than at parton level. This shows that the reconstruction and selection efficiencies decrease with increasing contribution of the anomalous couplings and it makes the determination of a small anomalous coupling contribution from a measurement of the total cross section difficult.

For the likelihood fit reference distributions of $\Delta \phi_{j j}$ after the detector simulation and event selection are calculated using reweighted events for 2000 equally spaced values of $g_{5 e}^{H Z Z}$ between -2 and 2 . In the regions between those values the histograms are linearly interpolated in each bin. Contributions by background processes are added to the signal distributions with the relative normalisation determined by the cross sections.

\subsection{Application to few individual pseudo-data samples}

The likelihood fit is performed on the same standard model Higgs boson pseudo-data samples that were used in section 5. For the $H \rightarrow \tau^{+} \tau^{-}$channels the likelihood is calculated in 5 bins. For the channel $H \rightarrow W^{+} W^{-} \rightarrow l l \nu \nu 10$ bins are used at an integrated luminosity of $10 \mathrm{fb}^{-1}$ and 20 bins are used at $30 \mathrm{fb}^{-1}$. Likelihood curves for one pseudo-data sample per channel and integrated luminosity studied are shown in figure 17. The results from the $H \rightarrow \tau^{+} \tau^{-}$channels are combined by adding the logarithms of the likelihoods. The minima of the likelihood curves and the 1-, 2- and $3 \sigma$ intervals are given in table 13 .

In the channel $H \rightarrow W^{+} W^{-} \rightarrow l l \nu \nu$ the estimated standard deviation of the parameter $g_{5 e}^{H Z Z}$ is 0.11 for an integrated luminosity of $30 \mathrm{fb}^{-1}$. As mentioned in section 2 the normalisation of $g_{5 e}^{H Z Z}$ is chosen such that a purely anomalous coupling of $g_{5 e}^{H W W}=g_{5 e}^{H Z Z} \cos ^{2} \theta_{w}=1$ roughly reproduces the standard model cross section in the calculation at $m_{H}=120 \mathrm{GeV}$. For a purely $\mathrm{CP}$ even anomalous coupling the same normalisation roughly reproduces the standard model cross section also at $m_{H}=160 \mathrm{GeV}$ in the channel $H \rightarrow W^{+} W^{-} \rightarrow l l \nu \nu$. According to the result for the standard deviation it should be possible to reduce the limits on a contribution of the anomalous $\mathrm{CP}$ even couplings for a standard model Higgs boson at $m_{H}=160 \mathrm{GeV}$ in the channel $H \rightarrow W^{+} W^{-} \rightarrow l l \nu \nu$ by roughly a factor of 6 with respect to the current limits from LEP and L3 given in table 3 which correspond approximately to a $95 \%$ confidence level or about $2 \sigma$. An improvement of the current limits should already be possible for an integrated luminosity of $10 \mathrm{fb}^{-1}$. In the $H \rightarrow \tau^{+} \tau^{-}$channels at $m_{H}=120 \mathrm{GeV}$ the expected standard deviation corresponds roughly to the current limits 

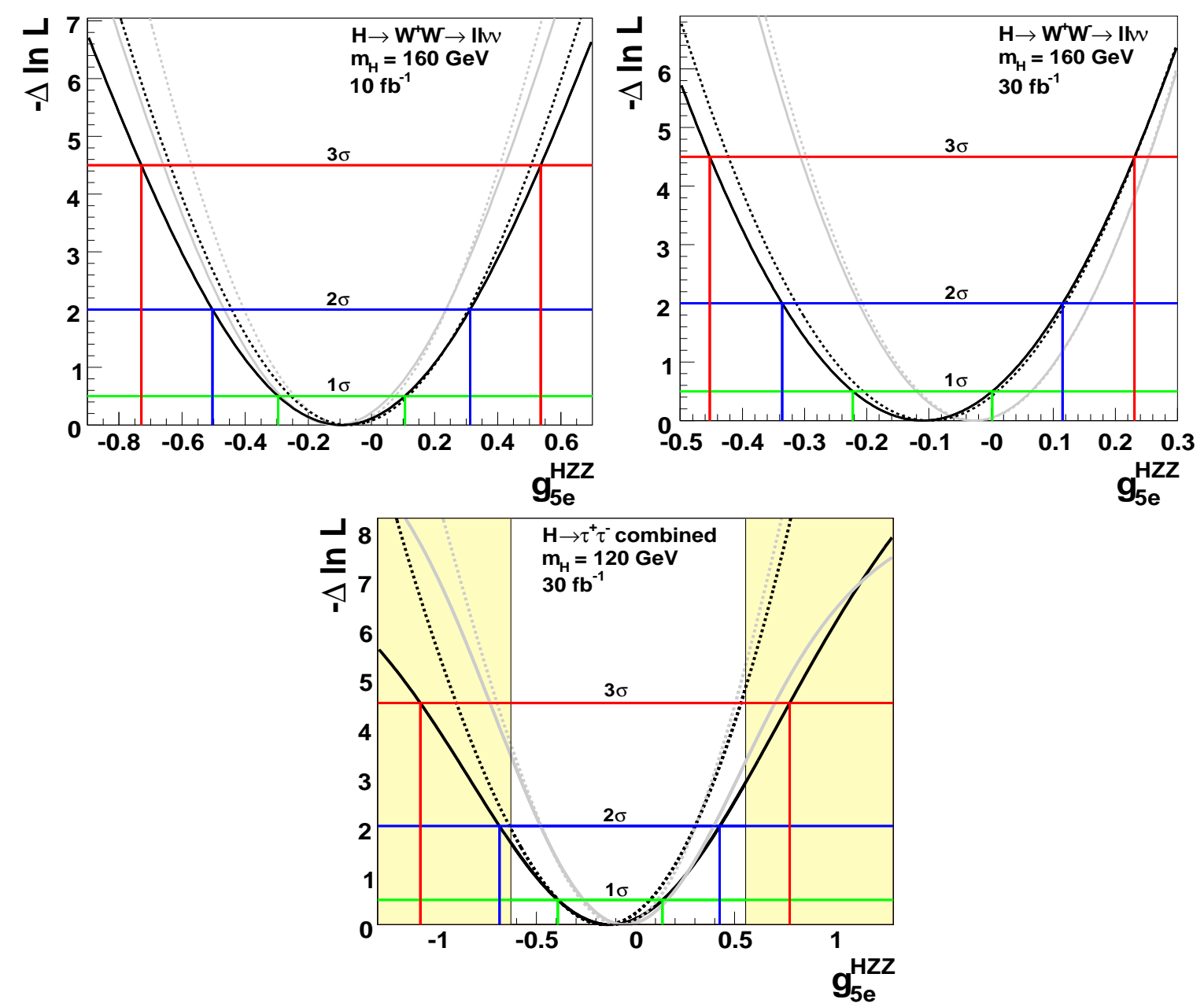

Figure 17: The logarithm of the likelihood as a function of the anomalous coupling constant $g_{5 e}^{H Z Z}$ for one pseudo-data sample per channel and integrated luminosity studied. The curves that were calculated with background contributions taken into account are shown in black. For comparison, curves that were calculated without considering background processes are shown in grey. The continuous lines were calculated using the non-extended likelihood. The dotted lines were calculated using the extended likelihood. The areas marked in light yellow (light grey) are excluded according to the approximate limits at $95 \%$ confidence level given in table 3. 1-, 2-, and $3 \sigma$ intervals are shown for the case of a non-extended likelihood with background contributions taken into account.

for the anomalous $\mathrm{CP}$ even $H Z Z$ couplings. It should be possible, however, to reduce the limits on the anomalous $H W W$ couplings in these channels. For the anomalous $H W W$ coupling the improvement with respect to the current limits will be larger in all cases since the current limits for this coupling are weaker and the predicted standard deviation for $g_{5 e}^{H W W}$ is smaller than that of $g_{5 e}^{H Z Z}$ by a factor of $\cos ^{2} \theta_{w}$. However, one should keep in mind that the limits from L3 were determined in single parameter analyses while in this study both the $H W W$ couplings and the $H Z Z$ couplings are varied at the same time.

One can see from table 13 that the inclusion of the prediction of the total cross section in an extended likelihood fit does not significantly increase the sensitivity in the channel $H \rightarrow W^{+} W^{-} \rightarrow l l \nu \nu$. The effect is larger in the $H \rightarrow \tau^{+} \tau^{-}$channels where a reduction of 
background included:

\begin{tabular}{|c|c|c|c|c|c|}
\hline & $\begin{array}{c}\operatorname{minimum} \\
-\Delta \ln L \\
\end{array}$ & $1 \sigma$ interval & $2 \sigma$ interval & $3 \sigma$ interval & $\sigma$ estimate \\
\hline \multicolumn{6}{|l|}{$\bar{H}$} \\
\hline \multirow{2}{*}{$\begin{array}{cc}10 \mathrm{fb}^{-1} & \text { non-extended } \\
\text { extended }\end{array}$} & -0.09 & {$[-0.30,0.11]$} & {$[-0.50,0.31]$} & {$[-0.73,0.54]$} & 0.20 \\
\hline & -0.07 & {$[-0.26,0.12]$} & {$[-0.44,0.31]$} & {$[-0$} & 0.19 \\
\hline \multirow{2}{*}{$\begin{array}{cc}30 \mathrm{fb}^{-1} & \begin{array}{c}\text { non-extended } \\
\text { extended }\end{array} \\
\end{array}$} & -0.11 & {$[-0.22,0.00]$} & {$[-0.34,0.12]$} & {$[-0.45,0.23]$} & 0.11 \\
\hline & -0.10 & {$[-0.21,0.01]$} & {$[-0.31,0$} & {$[-0.42,0.23]$} & 0.11 \\
\hline \multirow{3}{*}{$\begin{array}{c}H \rightarrow \tau^{+} \tau^{-} \rightarrow l l+4 \nu \\
30 \mathrm{fb}^{-1} \quad \text { non-extended } \\
\\
\quad \text { extended }\end{array}$} & & & & & \\
\hline & -0.25 & {$[-0$.} & {$[-1$.} & - & 0.38 \\
\hline & -0.25 & {$[-0$.} & {$[-0.97,0.40]$} & {$[-1.38,0.74]$} & 0.33 \\
\hline \multirow{3}{*}{$\begin{array}{l}H \rightarrow \tau^{+} \tau^{-} \text {combined } \\
30 \mathrm{fb}^{-1} \quad \text { non-extended } \\
\\
\quad \text { extended }\end{array}$} & & & & & \\
\hline & -0.13 & {$[-0.40,0.13]$} & {$[-0.69,0.42]$} & {$[-1.08,0.78]$} & 0.27 \\
\hline & -0.16 & {$[-0.40,0.06]$} & {$[-0.64,0.40]$} & {$[-0.90,0,53]$} & 0.23 \\
\hline
\end{tabular}

background not included, for comparison:

\begin{tabular}{|c|c|c|c|c|c|}
\hline & $\begin{array}{c}\text { minimum } \\
-\Delta \ln L\end{array}$ & $1 \sigma$ interval & $2 \sigma$ interval & $3 \sigma$ interval & $\sigma$ estimate \\
\hline \multicolumn{6}{|l|}{$H \rightarrow W^{+} W^{-} \rightarrow l l \nu \nu$} \\
\hline $10 \mathrm{fb}^{-1}$ non-extended & -0.11 & {$[-0.29,0.06]$} & {$[-0.47,0.24]$} & {$[-0.66,0.42]$} & 0.17 \\
\hline extended & -0.08 & {$[-0.24,0.07]$} & {$[-0.40,0.24]$} & {$[-0.57,0.41]$} & 0.16 \\
\hline $30 \mathrm{fb}^{-1}$ non-extended & -0.03 & {$[-0.12,0.06]$} & {$[-0.21,0.16]$} & {$[-0.31,0.25]$} & 0.09 \\
\hline extended & -0.03 & {$[-0.12,0.07]$} & {$[-0.21,0.16]$} & {$[-0.30,0.25]$} & 0.09 \\
\hline \multirow{3}{*}{$\begin{array}{l}H \rightarrow \tau^{+} \tau^{-} \rightarrow l l+4 \nu \\
30 \mathrm{fb}^{-1} \quad \text { non-extended } \\
\\
\quad \text { extended }\end{array}$} & & & & & \\
\hline & -0.27 & {$[-0.55,0.00]$} & {$[-0.89,0.27]$} & {$[-1.89,0.61]$} & 0.28 \\
\hline & -0.27 & {$[-0.54,-0.01]$} & {$[-0.86,0.26]$} & {$[-1.25,0.55]$} & 0.27 \\
\hline \multirow{2}{*}{$\begin{array}{l}H \rightarrow \tau^{+} \tau^{-} \text {combined } \\
30 \mathrm{fb}^{-1} \quad \text { non-extended } \\
\\
\quad \text { extended }\end{array}$} & & & & & \\
\hline & $\begin{array}{l}-0.00 \\
-0.09\end{array}$ & {$[-0.21,0.10]$} & {$[-0.40,0,40]$} & {$[-0.70,0.50]$} & $\begin{array}{l}0.21 \\
0.19\end{array}$ \\
\hline
\end{tabular}

Table 13: Results for the parameter $g_{5 e}^{H Z Z}$ from the likelihood fit for one pseudo-data sample per channel and integrated luminosity studied. Results that have been obtained with background processes taken into account are given in the upper table. Results that have been obtained without considering background contributions are given in the lower table for comparison. For the $H \rightarrow \tau^{+} \tau^{-}$case the results for the channel $H \rightarrow \tau^{+} \tau^{-} \rightarrow l l+4 \nu$ and for both $H \rightarrow \tau^{+} \tau^{-}$channels combined are given. The estimate of $\sigma$ is determined by dividing the width of the $1 \sigma$ interval by 2 .

the expected standard deviation by about $15 \%$ is observed.

A comparison of the values calculated with background processes taken into account to the values calculated for the signal process only shows that, as expected, the expected sensitivity is lower if background contributions are included. In the $H \rightarrow \tau^{+} \tau^{-}$channels the expected standard deviation increases by about $29 \%$ when background contributions are included. In the channel $H \rightarrow W^{+} W^{-} \rightarrow l l \nu \nu$ the predicted standard deviation increases by up to $22 \%$.

\subsection{Test of the method using many pseudo-data samples}

The properties of the likelihood fit are studied in more detail by applying it to all standard model Higgs boson pseudo-data samples that were used in section 5.3. In this way the 


\begin{tabular}{|c|c|c|}
\hline & \multicolumn{2}{|c|}{$\begin{array}{l}\text { distributions of the fit results for } g_{5 e}^{H Z Z} \\
\text { for pseudo-data samples from resampling }\end{array}$} \\
\hline & mean & $\sigma$ \\
\hline 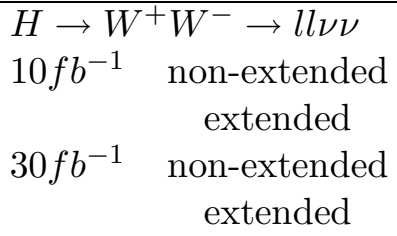 & $\begin{array}{r}(-0.8 \pm 2.2) \cdot 10^{-3} \\
(-0.7 \pm 2.2) \cdot 10^{-3} \\
(-0.1 \pm 1.1) \cdot 10^{-3} \\
(0.0 \pm 1.1) \cdot 10^{-3}\end{array}$ & $\begin{array}{c}0.1932 \pm 0.0021 \\
0.1921 \pm 0.0021 \\
0.11084 \pm 0.00080 \\
0.11039 \pm 0.00079\end{array}$ \\
\hline $\begin{array}{cc}H \rightarrow \tau^{+} \tau^{-} & \text {combined } \\
30 \mathrm{fb}^{-1} & \text { non-extended } \\
& \text { extended }\end{array}$ & $\begin{array}{r}(1.01 \pm 0.28) \cdot 10^{-2} \\
(-0.46 \pm 0.24) \cdot 10^{-2} \\
\end{array}$ & $\begin{array}{l}0.2612 \pm 0.0019 \\
0.2372 \pm 0.0017\end{array}$ \\
\hline
\end{tabular}

\begin{tabular}{|c|c|c|}
\hline & \multicolumn{2}{|c|}{$\begin{array}{c}\text { pull distributions } \\
\text { for pseudo-data samples from resampling }\end{array}$} \\
\hline & mean & $\sigma$ \\
\hline $\begin{array}{lc}H \rightarrow W^{+} W^{-} \rightarrow l l \nu \nu \\
10 \mathrm{fb}^{-1} & \text { non-extended } \\
& \text { extended } \\
30 \mathrm{fb}^{-1} \quad \text { non-extended } & \text { extended }\end{array}$ & $\begin{array}{c}(-2.0 \pm 0.97) \cdot 10^{-2} \\
(-1.96 \pm 0.99) \cdot 10^{-2} \\
(-1.0 \pm 1.0) \cdot 10^{-2} \\
(-1.1 \pm 1.0) \cdot 10^{-2}\end{array}$ & $\begin{array}{l}0.9728 \pm 0.0069 \\
0.9915 \pm 0.0070 \\
0.9954 \pm 0.0070 \\
1.0012 \pm 0.0071\end{array}$ \\
\hline $\begin{array}{l}H \rightarrow \tau^{+} \tau^{-} \text {combined } \\
30 \mathrm{fb}^{-1} \quad \text { non-extended } \\
\\
\quad \text { extended }\end{array}$ & $\begin{array}{r}(-1.66 \pm 0.89) \cdot 10^{-2} \\
(0.56 \pm 0.97) \cdot 10^{-2}\end{array}$ & $\begin{array}{l}0.8875 \pm 0.0063 \\
0.9727 \pm 0.0069\end{array}$ \\
\hline
\end{tabular}

Table 14: Results of Gaussian fits to the distributions of fit results for $g_{5 e}^{H Z Z}$ are given in the upper table. Results of Gaussian fits to the pull distributions are given in the lower table. The distributions were calculated by applying the likelihood fit to the pseudo-data samples from resampling as described in the text.

standard deviation of the estimated value of $g_{5 e}^{H Z Z}$ can be directly determined from the distribution of the fit results. Furthermore, the distribution of the fit results is used to study the possibility of a bias of the fit method. In addition to the distributions of the fit results pull distributions are calculated with a pull $z_{i}$ defined as the deviation of the fit result $m_{i}$ from the true value of $g_{5 e}^{H Z Z}$, which is 0 for the studied samples, to the standard deviation $\sigma\left(m_{i}\right)$ of the fit result as predicted from the width of the $-\Delta \ln L$ curve:

$$
z_{i}=\frac{m_{i}}{\sigma\left(m_{i}\right)}
$$

The pull distributions provide information about the correctness of the estimate of the standard deviation from the $-\Delta \ln L$ curves. If the estimated standard deviation on average is equal to the true standard deviation observed in the distribution of the fit results the pull distribution is expected to follow a normal Gaussian with mean 0 and standard deviation 1 ([26], p. 289f). If the pull distribution has a Gaussian shape with a standard deviation larger than 1 the error is on average underestimated from the width of the $-\Delta \ln L$ curve, if the standard deviation is smaller than 1 the error from the $-\Delta \ln L$ curve is on average overestimated.

Results are only presented for the pseudo-data samples from resampling as the results for disjoint subsamples are all consistent with the expectations and do not contain any additional 

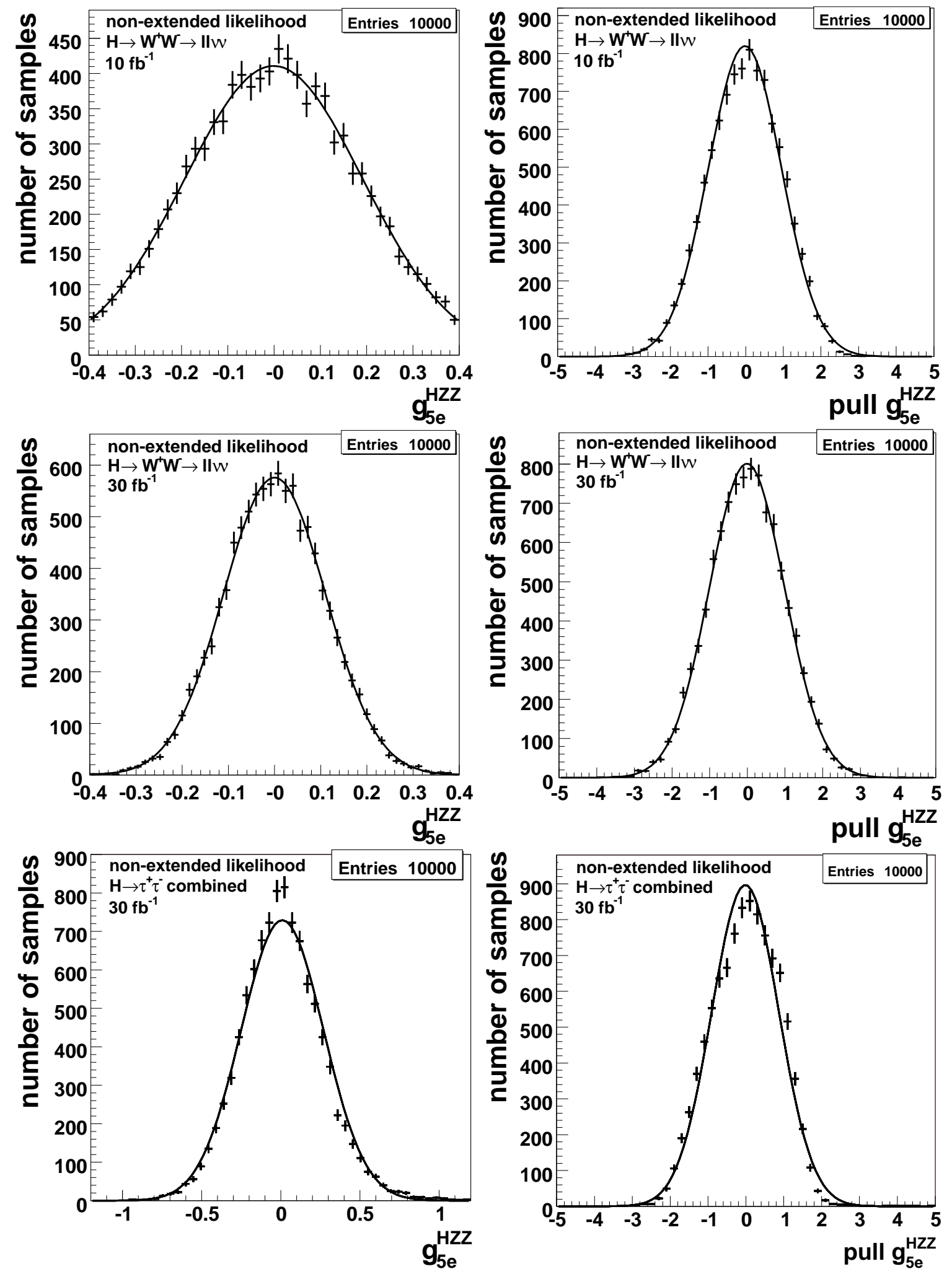

Figure 18: Distributions of the results of the non-extended likelihood fit for $g_{5 e}^{H Z Z}$ are shown on the left and the corresponding pull distributions are shown on the right. Gaussian functions that were fitted to all distributions are also shown. 

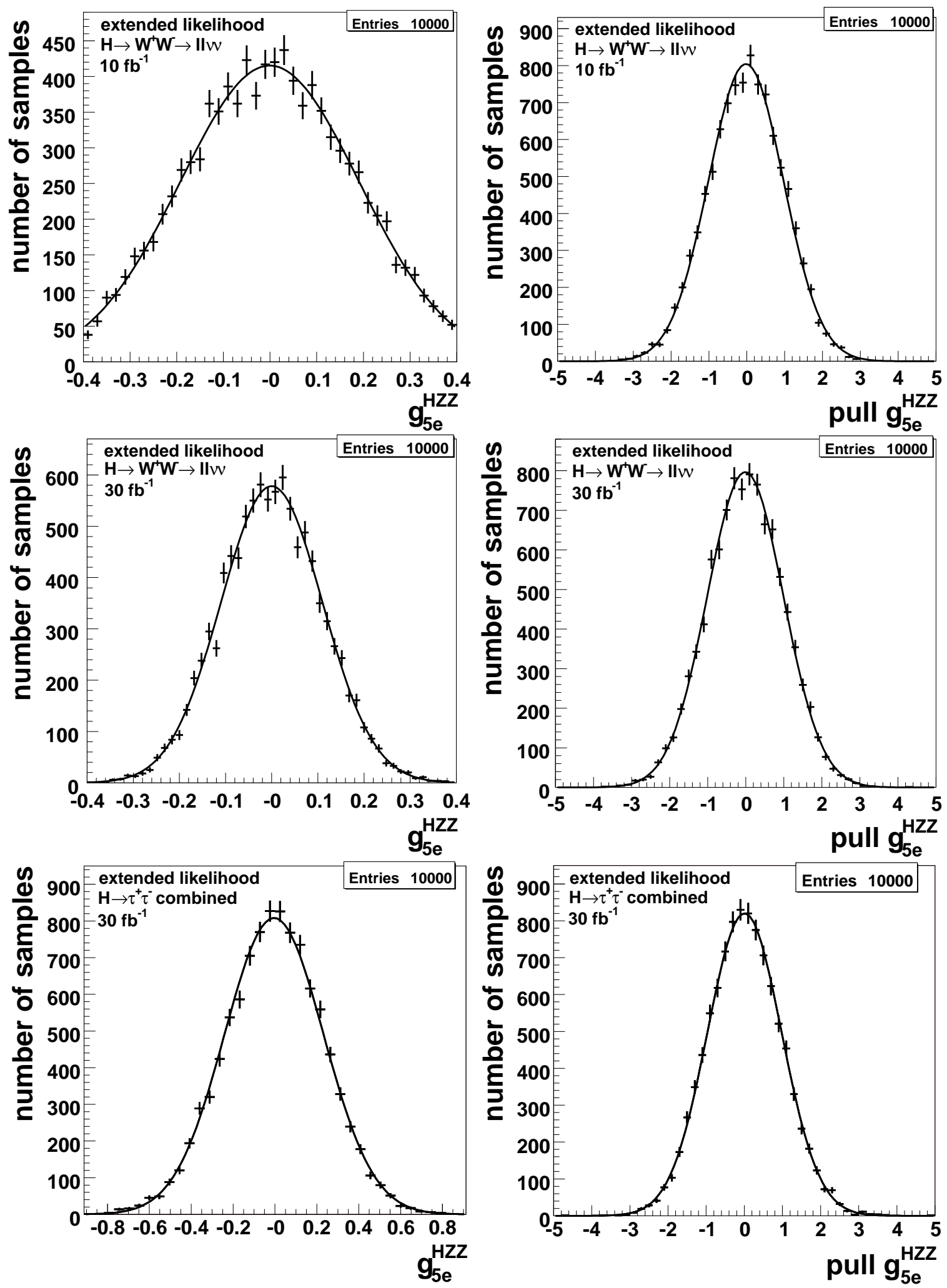

Figure 19: Distributions of the results of the extended likelihood fit for $g_{5 e}^{H Z Z}$ are shown on the left and the corresponding pull distributions are shown on the right. Gaussian functions that were fitted to all distributions are also shown. 
information. Distributions of the fit results for the anomalous coupling constant $g_{5 e}^{H Z Z}$ and pull distributions are shown in figure 18 for the non-extended likelihood and in figure 19 for the extended likelihood. Gaussian functions are fitted to all distributions and the results for the mean values and standard deviations of the Gaussians are given in table 14.

The results in table 14 for the standard deviation of the fit results for $g_{5 e}^{H Z Z}$ confirm the estimates from the individual likelihood fits in table 13. In the channel $H \rightarrow W^{+} W^{-} \rightarrow l l \nu \nu$ no statistically significant bias of the fit results is observed. In the $H \rightarrow \tau^{+} \tau^{-}$channels the results show small statistically significant biases which are attributed to deviations of the distributions from the Gaussian shape which can be seen in figure 18 and to a lesser degree in figure 19. The bias, while statistically significant, is smaller than $4 \%$ of the expected standard deviation in each case. The standard deviations of the pull distributions are compatible with 1 within statistical fluctuations for the channel $H \rightarrow W^{+} W^{-} \rightarrow l l \nu \nu$ and an integrated luminosity of $30 \mathrm{fb}^{-1}$. The standard deviations in this channel for $10 \mathrm{fb}^{-1}$ and in the $H \rightarrow \tau^{+} \tau^{-}$channels are slightly smaller than 1 which indicates that the errors are overestimated from the $-\Delta \ln L$ curves by a small factor in these cases.

\subsection{Effects of uncertainties on the number of background events}

The effects of an uncertainty on the measured number of background events of $\pm 10 \%$ on the results of the likelihood fit are studied as for the $\chi^{2}$ test (see section 5.4) by repeating the likelihood fit on pseudo-data samples in which the average number of events for all background processes is varied by $+10 \%$ and repeating it again for pseudo-data samples in which the average number of events for all background processes is varied by $-10 \%$.

One can see from the results given in tables 15 and 16 that a variation of the number of background events introduces a bias in the fit and a small change in the standard deviation of the fit results. The bias is larger than the change in the standard deviation in most cases and of similar size in others. The bias is negative if there are more background events in the data than assumed and positive if there are less background events.

For the $W \rightarrow W^{+} W^{-} \rightarrow l l \nu \nu$ channel the bias in the extended likelihood fit is larger than in the non-extended likelihood fit whereas for the $H \rightarrow \tau^{+} \tau^{-}$channels the bias in the non-extended likelihood fit is larger. The total uncertainty on the fit result for $g_{5 e}^{H Z Z}$ for a single data sample induced by a variation of the number of background events of $\pm 10 \%$ is approximately \pm 0.02 .

\section{Summary}

The prospects for the measurement of the structure of the Higgs boson coupling to two weak gauge bosons in the weak boson fusion channel are studied using the fast simulation of the ATLAS detector. The distribution of the azimuthal angles between the tagging jets $\Delta \phi_{j j}$ after an event selection optimised for maximum discovery potential is used in the analysis which is performed for the Higgs boson decay channels $H \rightarrow \tau^{+} \tau^{-} \rightarrow l l+4 \nu$ and $H \rightarrow \tau^{+} \tau^{-} \rightarrow l h+3 \nu$ at $m_{H}=120 \mathrm{GeV}$ and $H \rightarrow W^{+} W^{-} \rightarrow l l \nu \nu$ at $m_{H}=160 \mathrm{GeV}$.

In addition to the standard model vertex which is proportional to $g^{\mu \nu}$ additional tensor vertices which follow from terms of dimension 6 in an effective Lagrangian are studied. The coupling terms differ in their properties under CP transformations. Under the assumption of $\mathrm{CP}$ invariance the determination of the dominant coupling term permits the determination of the $\mathrm{CP}$ quantum number of the Higgs boson. The ratio of anomalous $H W W$ couplings to anomalous $H Z Z$ couplings was fixed for simplicity to $g_{5 e}^{H W W} / g_{5 e}^{H Z Z}=\cos ^{2} \theta_{w}$. It is shown that it should be possible with the ATLAS detector to exclude the possibility of a 
Results for $+10 \%$ background events:

\begin{tabular}{|c|c|c|}
\hline & \multicolumn{2}{|c|}{$\begin{array}{l}\text { distributions of the fit results for } g_{5 e}^{H Z Z} \\
\text { for pseudo-data samples from resampling }\end{array}$} \\
\hline & mean & $\bar{\sigma}$ \\
\hline 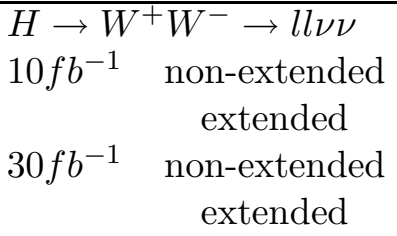 & $\begin{array}{c}(-8.1 \pm 2.1) \cdot 10^{-3} \\
(-1.15 \pm 0.22) \cdot 10^{-2} \\
(-6.2 \pm 1.1) \cdot 10^{-3} \\
(-9.7 \pm 1.1) \cdot 10^{-3}\end{array}$ & $\begin{array}{c}0.1872 \pm 0.0019 \\
0.1927 \pm 0.0021 \\
0.11000 \pm 0.00079 \\
0.11360 \pm 0.00082\end{array}$ \\
\hline $\begin{array}{l}H \rightarrow \tau^{+} \tau^{-} \text {combined } \\
30 \mathrm{fb}^{-1} \quad \text { non-extended } \\
\\
\quad \text { extended }\end{array}$ & $\begin{array}{c}(-9.3 \pm 2.6) \cdot 10^{-3} \\
(-1.00 \pm 0.26) \cdot 10^{-2}\end{array}$ & $\begin{array}{l}0.2594 \pm 0.0018 \\
0.2557 \pm 0.0018\end{array}$ \\
\hline
\end{tabular}

\begin{tabular}{|c|c|c|}
\hline & \multicolumn{2}{|c|}{$\begin{array}{c}\text { pull distributions } \\
\text { for pseudo-data samples from resampling }\end{array}$} \\
\hline & mean & $\sigma$ \\
\hline $\begin{array}{lc}H \rightarrow W^{+} W^{-} \rightarrow l l \nu \nu \\
10 \mathrm{fb}^{-1} & \text { non-extended } \\
& \text { extended } \\
30 \mathrm{fb}^{-1} \quad \begin{array}{c}\text { non-extended } \\
\text { extended }\end{array} \\
\end{array}$ & $\begin{array}{c}(-4.89 \pm 0.96) \cdot 10^{-2} \\
(-6.5 \pm 1.0) \cdot 10^{-2} \\
(-6.5 \pm 1.0) \cdot 10^{-2} \\
(-9.8 \pm 1.0) \cdot 10^{-2} \\
\end{array}$ & $\begin{array}{l}0.9642 \pm 0.0068 \\
1.0006 \pm 0.0071 \\
1.0015 \pm 0.0071 \\
1.0256 \pm 0.0073\end{array}$ \\
\hline $\begin{array}{l}H \rightarrow \tau^{+} \tau^{-} \text {combined } \\
30 \mathrm{fb}^{-1} \quad \text { non-extended } \\
\\
\quad \text { extended }\end{array}$ & $\begin{array}{c}(-8.53 \pm 0.91) \cdot 10^{-2} \\
(-1.0 \pm 1.0) \cdot 10^{-2}\end{array}$ & $\begin{array}{l}0.9051 \pm 0.0064 \\
1.0150 \pm 0.0072\end{array}$ \\
\hline
\end{tabular}

Table 15: Results of the likelihood fit to pseudo-data samples from resampling with the average number of events for all background processes varied by $+10 \%$ with respect to the expected number. Results of Gaussian fits to the distributions of fit results for $g_{5 e}^{H Z Z}$ are given in the upper table. Results of Gaussian fits to the pull distributions are given in the lower table.

purely anomalous CP even or a purely anomalous CP odd coupling at a confidence level corresponding to approximately $5 \sigma$ for a standard model Higgs boson at $m_{H}=160 \mathrm{GeV}$ from the analysis in the channel $H \rightarrow W^{+} W^{-} \rightarrow l l \nu \nu$ for low luminosity data corresponding to an integrated luminosity of $10 \mathrm{fb}^{-1}$. For a standard model Higgs boson at $m_{H}=120 \mathrm{GeV}$ an exclusion with a confidence level corresponding to $2 \sigma$ or more is considered possible from the analysis in the $H \rightarrow \tau^{+} \tau^{-}$channels for an integrated luminosity of $30 \mathrm{fb}^{-1}$. If the confidence level observed for one anomalous coupling (CP even or CP odd) is lower than the stated values it is expected that the confidence level for the other anomalous coupling will be higher. An uncertainty of $\pm 10 \%$ on the measured number of background events is shown to have only a small effect on the expected exclusion limits.

The sensitivity of the ATLAS experiment to a small anomalous CP even coupling present in addition to the standard model coupling is studied using a likelihood fit. The standard deviation of the measured anomalous coupling constant $g_{5 e}^{H Z Z}$ for the case of a standard model Higgs boson at $m_{H}=160 \mathrm{GeV}$ in the channel $H \rightarrow W^{+} W^{-} \rightarrow l l \nu \nu$ is estimated to be 0.11 for an integrated luminosity of $30 \mathrm{fb}^{-1}$ and 0.19 for an integrated luminosity of $10 \mathrm{fb}^{-1}$. The normalisation of $g_{5 e}^{H Z Z}$ is chosen such that a value for $g_{5 e}^{H Z Z}$ of $1 / \cos ^{2} \theta_{w}$ roughly gives the standard model cross section in a calculation with a purely anomalous coupling. A 
Results for $-10 \%$ background events:

\begin{tabular}{|c|c|c|}
\hline & \multicolumn{2}{|c|}{$\begin{array}{l}\text { distributions of the fit results for } g_{5 e}^{H Z Z} \\
\text { for pseudo-data samples from resampling }\end{array}$} \\
\hline & mean & $\bar{\sigma}$ \\
\hline \multirow{5}{*}{$\begin{array}{lc}H \rightarrow W^{+} W^{-} \rightarrow l l \nu \nu \\
10 \mathrm{fb}^{-1} \quad \text { non-extended } \\
& \text { extended } \\
30 \mathrm{fb}^{-1} \quad \text { non-extended } \\
& \text { extended }\end{array}$} & & \\
\hline & $(4.6 \pm 2.2) \cdot 10^{-3}$ & $0.1909 \pm 0.0019$ \\
\hline & $(8.4 \pm 2.1) \cdot 10^{-3}$ & $0.1844 \pm 0.0019$ \\
\hline & $(5.5 \pm 1.1) \cdot 10^{-3}$ & $0.11279 \pm 0.00081$ \\
\hline & $(9.4 \pm 1.1) \cdot 10^{-3}$ & $0.10814 \pm 0.00077$ \\
\hline \multirow{3}{*}{$\begin{array}{l}H \rightarrow \tau^{+} \tau^{-} \text {combined } \\
30 \mathrm{fb}^{-1} \quad \text { non-extended } \\
\\
\quad \text { extended }\end{array}$} & & \\
\hline & $(2.84 \pm 0.27) \cdot 10^{-2}$ & $0.2686 \pm 0.0019$ \\
\hline & $(-1.6 \pm 2.3) \cdot 10^{-3}$ & $0.2313 \pm 0.0016$ \\
\hline
\end{tabular}

\begin{tabular}{|c|c|c|}
\hline & \multicolumn{2}{|c|}{$\begin{array}{c}\text { pull distributions } \\
\text { for pseudo-data samples from resampling }\end{array}$} \\
\hline & mean & $\sigma$ \\
\hline $\begin{array}{lc}H \rightarrow W^{+} W^{-} \rightarrow l l \nu \nu \\
10 \mathrm{fb}^{-1} & \text { non-extended } \\
& \text { extended } \\
30 \mathrm{fb}^{-1} & \text { non-extended } \\
& \text { extended } \\
& \end{array}$ & $\begin{array}{c}(1.31 \pm 0.96) \cdot 10^{-2} \\
(3.38 \pm 0.96) \cdot 10^{-2} \\
(4.0 \pm 1.0) \cdot 10^{-2} \\
(7.50 \pm 0.99) \cdot 10^{-2}\end{array}$ & $\begin{array}{l}0.9565 \pm 0.0068 \\
0.9601 \pm 0.0068 \\
0.9961 \pm 0.0070 \\
0.9854 \pm 0.0070\end{array}$ \\
\hline $\begin{array}{l}H \rightarrow \tau^{+} \tau^{-} \text {combined } \\
30 \mathrm{fb}^{-1} \quad \text { non-extended } \\
\\
\quad \text { extended }\end{array}$ & $\begin{array}{l}(4.33 \pm 0.89) \cdot 10^{-2} \\
(1.50 \pm 0.96) \cdot 10^{-2}\end{array}$ & $\begin{array}{l}0.8917 \pm 0.0064 \\
0.9613 \pm 0.0068\end{array}$ \\
\hline
\end{tabular}

Table 16: Results of the likelihood fit to pseudo-data samples from resampling with the average number of events for all background processes varied by $-10 \%$ with respect to the expected number. Results of Gaussian fits to the distributions of fit results for $g_{5 e}^{H Z Z}$ are given in the upper table. Results of Gaussian fits to the pull distributions are given in the lower table.

measurement of a contribution of an anomalous coupling of 0 and a standard deviation as estimated for an integrated luminosity of $30 \mathrm{fb}^{-1}$ would correspond to an improvement of the current experimental limits from measurements at LEP and especially the L3 experiment by roughly a factor of 6 . The results given were determined using only the shape of the $\Delta \phi_{j j}$ distribution. The inclusion of the prediction of the total cross section in an extended likelihood fit gives practically no advantage. No statistically significant bias is observed in distributions of the fit results for many Monte Carlo event samples.

For a standard model Higgs boson at $m_{H}=120 \mathrm{GeV}$ in the $H \rightarrow \tau^{+} \tau^{-}$channels a standard deviation of 0.26 from a non-extended likelihood fit using the shape of the $\Delta \phi_{j j}$ distribution alone and 0.24 from an extended likelihood fit is expected for an integrated luminosity of $30 \mathrm{fb}^{-1}$. For the anomalous $H Z Z$ coupling this result corresponds roughly to the current experimental limits while it would permit an improvement of the limits for the anomalous $H W W$ coupling. Distributions of the fit results show small deviations from the Gaussian shape and a small statistically significant bias is observed in the fit results for these channels.

It is shown that an uncertainty of $\pm 10 \%$ on the number of background events in the data leads to an uncertainty of roughly \pm 0.02 on the fit results in both decay channels of the Higgs boson. 


\subsection{Concluding Remarks}

The effects of the emission of an additional gluon on the $\Delta \phi_{j j}$ distribution in the weak boson fusion process have been studied in [5]. It was found that the changes to the distribution in a next-to-leading order QCD calculation are very small and no significant effect on the results of this study is expected from those corrections to the signal process.

Recently it has been shown [24] that it is possible to observe interference effects between CP even and CP odd couplings by using a different definition of the azimuthal angle between the tagging jets. Instead of using the absolute value of the angle, as was done in this study, the sign of the angle is defined with respect to the direction of the tagging jet in one arbitrarily fixed detector hemisphere. The prospects for the measurement with the ATLAS detector of a CP odd coupling contribution in addition to standard model and/or CP even anomalous couplings using the redefined azimuthal angle difference are possible topics for further research.

Version 10.0.1 of ATHENA was used. The version of ATLFAST used in ATHENA 10.0.1 has not been formally validated within the ATLAS collaboration and, albeit being considered as appropriate for this study, results obtained using this version cannot be compared directly to other ATLAS results.

\section{Acknowledgements}

We would like to thank Dieter Zeppenfeld for providing the program vbfnlo as well as Dieter Zeppenfeld and Vera Hankele for some very helpful comments and discussions.

This work has been performed within the ATLAS Collaboration, and we thank collaboration members for helpful discussions. We have made use of the ATLAS physics analysis framework and tools which are the result of collaboration-wide efforts.

\section{References}

[1] S.Asai et al., Prospects for the Search for a Standard Model Higgs Boson in ATLAS using Vector Boson Fusion, SN-ATLAS-2003-024, Eur. Phys. J. C32 S2 (2004) 19

[2] T. Plehn, D. Rainwater, D. Zeppenfeld, Determining the Structure of Higgs Couplings at the CERN Large Hadron Collider, Phys. Rev. Lett. 88, (2002) 051801, hep-ph/0105325

[3] C. Ruwiedel, Studie zur Messbarkeit der Struktur der Kopplung eines Higgs-Bosons an schwache Eichbosonen in der Vektorbosonfusion mit dem ATLAS-Detektor am LHC, Diplomarbeit, BONN-IB-2006-06

[4] W. Buchmüller, D. Wyler, Effective Lagrangian analysis of new interactions and flavour conservation, Nucl. Phys. B268 (1986) 621

[5] T. Figy, D. Zeppenfeld, QCD Corrections to Jet Correlations in Weak Boson Fusion, Phys. Lett. B591 (2004) 297, hep-ph/0403297

[6] The L3 Collaboration, P. Achard et al., Search for Anomalous Couplings in the Higgs Sector at LEP, Phys. Lett. B589 (2004) 89, hep-ex/0403037

[7] E. Boos et al., Generic User Process Interface for Event Generators, hep-ph/0109068

[8] T. Sjöstrand et al., High-Energy-Physics Event Generation with PyтHia 6.1, Comp. Phys. Commun. 135 (2001) 238, hep-ph/0010017 
[9] S. Jadach, Z. Was, R. Decker, J.H. Kuehn, The tau decay library TAUOLA: version 2.4, Comp. Phys. Commun. 76 (1993) 361

[10] E. Barberio, Z. Was, PHOTOS-A universal Monte Carlo for QED radiative corrections: version 2.0, Comp. Phys. Commun. 79 (1994) 291

[11] E. Richter-Was, D. Froidevaux, L. Poggioli, ATLFAST 2.0, a fast simulation package for ATLAS, ATL-PHYS-98-131

[12] H.L. Lai et al., Global QCD analysis of parton structure of the nucleon: CTEQ5 parton distributions, Eur. Phys. J. C12 (2000) 375, hep-ph/9903282

[13] D. Zeppenfeld et al., provided by the auther via e-mail

[14] D. Zeppenfeld, private communication

[15] S. R. Slabospitsky, L. Sonnenschein, TopReX generator (version 3.25) short manual, Comput. Phys. Commun. 148 (2002) 87, hep-ph/0201292

[16] F. Maltoni, T. Stelzer, MadEvent: Automatic event generation with MadGraph, JHEP 0302:027 (2003), hep-ph/0208156

[17] H. Hagiwara, D. Zeppenfeld, Amplitudes for multi-parton processes involving a current at $e^{+} e^{-}, e^{ \pm} p$, and hadron colliders, Nucl. Phys. B313 (1989) 560

[18] H. Chehime, D. Zeppenfeld, Single $W$ - and Z-boson production as a probe for rapidity gaps at the Superconducting Super Collider, Phys. Rev. D47 (1993) 3898

[19] Particle Data Group, Particle Physics Booklet; from S. Eidelman et al., Review of Particle Physics, Phys. Lett. B592 (2004) 1

[20] ATLAS Collaboration, O.B. Abdinov et al., ATLAS detector and physics performance, Technical Design Report, CERN/LHCC/99-14 and CERN/LHCC/99-15

[21] Michael Heldmann, private communication

[22] R. Barlow, Resampling and the Bootstrap, SLUO Lecture on Statistics and Numerical Methods in HEP, http://www.hep.man.ac.uk/u/roger/

[23] The LEP Collaborations ALEPH, DELPHI, L3, OPAL, and the LEP Electroweak Working Group, A Combination of Preliminary Electroweak Measurements and Constraints on the Standard Model, hep-ex/0511027

[24] V. Hankele, G. Klämke, D. Zeppenfeld, T. Figy, Anomalous Higgs boson couplings in vector boson fusion at the CERN LHC, hep-ph/0609075

[25] R. Barlow, Event Classification Using Weighting Methods, J. Comp. Phys. 72 (1987) 202

[26] A. G. Frodesen, O. Skjeggestad, H. Tøfte, Probability and Statistics in Particle Physics, Universitetsforlaget, Oslo (1979) 